\title{
LMI-Based Robust Stabilization of a Class of Input-Constrained Uncertain Nonlinear Systems with Application to a Helicopter Model
}

\author{
Hassène Gritli (iD ${ }^{1,2}$ \\ ${ }^{1}$ Institut Supérieur des Technologies de l'Information et de la Communication, Université de Carthage, 1164 Borj Cedria, \\ Tunis, Tunisia \\ ${ }^{2}$ Laboratoire Robotique, Informatique et Systèmes Complexes (RISC-LR16ES07), Ecole Nationale d'Ingénieurs de Tunis, \\ Université de Tunis El Manar, BP. 37, Le Belvédère, 1002 Tunis, Tunisia
}

Correspondence should be addressed to Hassène Gritli; grhass@yahoo.fr

Received 22 August 2019; Revised 21 November 2019; Accepted 10 December 2019; Published 25 January 2020

Guest Editor: Baltazar Aguirre-Hernandez

Copyright (c) 2020 Hassène Gritli. This is an open access article distributed under the Creative Commons Attribution License, which permits unrestricted use, distribution, and reproduction in any medium, provided the original work is properly cited.

\begin{abstract}
This paper is concerned with the robust stabilization of a class of continuous-time nonlinear systems, with an application to the pitch dynamics of a simple helicopter model, via an affine state-feedback control law using the linear matrix inequality (LMI) approach. The nonlinear dynamics is subject to norm-bounded parametric uncertainties and disturbances. In addition, the problem of actuator nonlinearity is addressed by considering the saturation effect of the control law. We demonstrate first that the synthesis problem of the saturated controller is expressed in terms of bilinear matrix inequalities (BMIs). Thanks to the Schur complement lemma and the matrix inversion lemma, we convert these BMIs into LMIs allowing the simultaneous computation of the two gains of the affine controller. Furthermore, we address in this work the estimation problem of the domain of attraction using the invariant set concept. This is solved by computing the largest attractive invariant ellipsoid. Compared with previous works, the research procedure of such ellipsoidal set is achieved in a single step with a reduced number of LMI constraints and then with less conservative conditions. A portfolio of numerical results is presented. The effectiveness and robustness of the proposed saturated controller in the stabilization of the adopted helicopter pitch model toward parametric uncertainties and disturbances are illustrated through simulation results.
\end{abstract}

\section{Introduction}

1.1. Background and Literature Review. Mechatronics, such as aircraft, spacecrafts, launch vehicles, unmanned autonomous vehicles, missiles, walking robots, robot manipulators, electronic vehicles, and unmanned aerial vehicles, has played a very important role in modern industry-related applications. Nowadays, there is an ever-increasing demand of advanced control strategies for mechatronic systems with enhanced performances.

It is known, on the one hand, that almost all existing physical and mechatronic systems unavoidably include uncertainties and disturbances due to inaccurate modeling, measurement errors, exterior conditions, or parameter variations. The presence of uncertainties may cause instability and bad performances on a controlled system. Thus, considerable efforts have been assigned to the robust stability and stabilization of linear and nonlinear systems with parametric uncertainties. For a recent literature, we refer the readers to [1-8]. Two types of parametric uncertainties are very often considered in systems: norm-bounded uncertainty and polytopic uncertainty. In recent years, the linear matrix inequality (LMI) technique [9] has been widely used to solve the robust control for uncertain linear and nonlinear systems with polytopic uncertain parameters and norm-bounded uncertain parameters. However, most control synthesis problems cannot be written in a LMI form. However, they are written in terms of a more general form known as a bilinear matrix inequality (BMI), which is usually not exploitable numerically to solve. For some BMI 
problems with simple bilinearities, the YALMIP toolbox [10] was used to solve such problems. There are several approaches and different relaxed synthesis conditions proposed for conducting the BMIs into LMIs, such as in [5-7, 9, 11-16], just to mention a few.

On the other hand, the actuator saturation nonlinearity exists commonly in practical control systems. Indeed, the signal amplitude that an actuator can deliver is usually limited by physical or safety constraints. Thus, the neglect of the actuator constraints in the controller design may cause instability of the closed-loop system [17-19]. The design of controllers for continuous-time and discrete-time systems by taking into consideration the actuator saturation has been extensively studied in the past few decades [17-36]. Some works have investigated the problem of uncertainties and actuator saturation in mechatronic systems such as the vehicle lateral dynamics [37], the vehicle active suspension systems [38-41], the rigid spacecraft $[42,43]$, the inertia wheel inverted pendulum [12], the supercavitating vehicle [44], the flexible robotic manipulator [45], and the marine surface vessel [46], among others.

The concept of attractive invariant sets has been generally proposed for linear systems involving actuator saturation $[5,20,28,29,36,44,47-51]$. Moreover, through such concept, numerous studies have focused on the characterization of the maximum attraction set for linear systems subject to saturated linear feedback. Thus, some research works focused on writing the saturation function of the control input as a linear convex combination of some constrained variables $[18,20,27,36,47,48,50-53]$. This approach leads to sufficient conditions in terms of a very large number of LMIs that are difficult to solve numerically [27-29]. The common goal of almost all of these previous research works is to find the largest attractive invariant ellipsoidal set in order to provide an estimation of the domain of attraction for initial conditions.

In addition, it is well recognized that most practical control systems are inherently nonlinear. The number of available results by taking the actuator saturation nonlinearity into account in the design and analysis of nonlinear control systems is still limited. Because of the difficulty of the problem itself, most research works have been focused on particular classes of nonlinear systems to design saturated controllers $[5,25,26]$. Furthermore, the simultaneous presence of parametric uncertainties and actuator saturation nonlinearity in physical systems has led many authors to combine the techniques of the robust control theory and those of constrained control $[5,54]$.

1.2. Objective of the Paper. In this paper, we are interested in the design of a robust controller to stabilize a class of nonlinear systems, for which the zero state is not the equilibrium point. As a motivation application, we consider the pitch dynamics of a simple helicopter model [55-57]. In fact, such helicopter pitch model has been considered as a testbed used in order to develop new advanced control strategies. Authors in [56] approximated the pitch dynamics of the helicopter with a set of piecewise affine stochastic systems. The proposed stochastic affine feedback controller is designed for the approximated model and implemented on the main nonlinear system. Authors in [57] designed an observer-based minimum variance control for the same objective by taking into consideration norm-bounded uncertainties. Such uncertainties arise in the form of the difference between the actual nonlinear dynamics and its piecewise-affine approximation. Furthermore, authors in [55] approximated also the simplified pitch model of the helicopter via a piecewise affine system. In these works [55-57], the saturation effect in the actuators was not taken into consideration in the control design.

In the present work, we propose an affine state-feedback control law for the adopted class of nonlinear systems, with a particular interest to the pitch dynamics of the helicopter model, where all the states are assumed to be available for direct measurement. Our main objective is to control, via such affine feedback control law, the adopted nonlinear systems to the zero state. Moreover, we consider the problem of norm-bounded parametric uncertainties and also the problem of external disturbances. Furthermore, we take into consideration the problem of actuator saturation in the design of the robust affine state-feedback control law. Thus, the main role of such control law is to asymptotically bring the trajectory of the nonlinear system to the zero state even if it is subject to the parametric uncertainties, the disturbances, and the actuator saturation. In addition, in this work, the problem of searching for appropriate feedback gain matrices of the affine state-feedback control law is realized also by solving the problem of estimating the largest attractive invariant set. Our design methodology of the saturated stabilizing affine state-feedback control law is based on the framework of LMIs. We show at first that the affine statefeedback controller is designed by solving an optimization problem subject to three BMI constraints. Then, by using some judicious congruence transformations and some technical lemmas, we convert these BMIs into three LMI constraints. In the end of this work, we demonstrate through simulations the effectiveness of the proposed affine statefeedback control law in the robust stabilization of the pitch dynamics of the helicopter model while the largest attractive invariant set is guaranteed.

1.3. Contributions and Innovations. The main contributions and innovations of the work in this paper can be summarized as follows:

(1) The problem of robust stabilization of a class of nonlinear systems, with an application to the pitch dynamics of a simple helicopter model, under normbounded parametric uncertainties, external disturbance, and input saturation nonlinearity, is considered using the concept of the invariant set. The main objective is to control the disturbed uncertain nonlinear system to the zero state, which is not the equilibrium point in the open loop.

(2) A saturated affine state-feedback controller is designed based on the LMI approach. To the best of 
the authors' knowledge, such problem was not developed in the literature. Only the linear state/output feedback controllers have been considered.

(3) A transformation of the BMI stability condition, related to the input saturation problem, into an LMI condition is achieved using the matrix inversion lemma and the Schur complement lemma.

(4) An estimation of the ellipsoidal region of attraction for the nonlinear system under study is also realized. Compared with previous works [20,47-50, 52, 58], our research procedure of the largest invariant attractive ellipsoid is achieved in only one step with a reduced number of LMI constraints.

1.4. Structure of the Paper. The rest of this paper is organized as follows. In Section 2, we present first some technical lemmas that will be used in this work, the adopted class of the nonlinear systems, and the problem formulation. The simple helicopter model, as a motivation application, and its pitch dynamics are also described in this section. The design of the robust affine state-feedback controller under saturation, based on the LMI approach, is discussed in Section 3. Transformation of the BMIs into LMIs is also realized in this section. The problem of computation/estimation of the domain of attraction (the largest attractive ellipsoid) is addressed in Section 4. Simulation results and some comparisons are presented in Section 5. Finally, concluding remarks and future works are drawn in Section 6.

1.5. Notations. Throughout this paper, $\mathbf{A}^{\mathrm{T}}$ represents the transpose of $\mathbf{A}$, the symbol $(*)$ in matrix inequality denotes the symmetric term of the matrix, for example, $\left[\begin{array}{cc}\mathbf{X} & \mathbf{Y} \\ (*) & \mathbf{Z}\end{array}\right]=$ $\left[\begin{array}{cc}\mathbf{X} & \mathbf{Y} \\ \mathbf{Y}^{\mathrm{T}} & \mathbf{Z}\end{array}\right]$ and $\mathbf{X}+(*)=\mathbf{X}+\mathbf{X}^{\mathrm{T}}, \mathbf{X}>0(<0)$ means $\mathbf{X}$ is a symmetric positive (negative) definite matrix, and $\operatorname{diag}(A, B, \ldots, Z)$ represents a diagonal matrix. Moreover, $\mathcal{O}$ and $\mathscr{I}$ denote the zero matrix and the identity matrix, respectively, with appropriate dimensions.

\section{Preliminaries and Problem Statement}

In this section, we define the class of continuous-time nonlinear systems that will be investigated in this paper. The pitch dynamics of a simple helicopter model is given as an illustrative example. First of all, we present some technical lemmas that will be used subsequently.

\subsection{Some Technical Lemmas}

Lemma 1 (see [41]). The Lyapunov candidate function $V(t)$ is bounded given that the initial condition $V(0)$ is bounded, $V(t) \geq 0$ is continuous, and if the following equation is true:

$$
\dot{V}(t) \leq-\mu V(t)+\eta,
$$

where $\mu>0$ and $\eta>0$.
Lemma 2 (the Young relation [12]). Given constant matrices $\mathbf{X}$ and $\mathbf{Y}$ with appropriate dimensions, the following inequality holds:

$$
\mathbf{X}^{\mathrm{T}} \mathbf{Y}+\mathbf{Y}^{\mathrm{T}} \mathbf{X} \leq \varepsilon \mathbf{X}^{\mathrm{T}} \mathbf{X}+\varepsilon^{-1} \mathbf{Y}^{\mathrm{T}} \mathbf{Y}
$$

for all positive scalar $\varepsilon$.

Lemma 3 (the matrix inversion lemma $[9,12])$. Given invertible matrices $\mathbf{A}$ and $\mathbf{B}$ such that $\mathbf{A} \in \mathbb{R}^{n \times n}$ and $\mathbf{B} \in \mathbb{R}^{m \times m}$. Moreover, given matrices $\mathbf{C}$ and $\mathbf{D}$ with appropriate dimensions: $\mathbf{C} \in \mathbb{R}^{n \times m}$ and $\mathbf{D} \in \mathbb{R}^{m \times n}$. Then,

$$
(\mathbf{A}+\mathbf{C B D})^{-1}=\mathbf{A}^{-1}-\mathbf{A}^{-1} \mathbf{C}\left(\mathbf{B}^{-1}+\mathbf{D A}^{-1} \mathbf{C}\right)^{-1} \mathbf{D A}^{-1} \text {. }
$$

Lemma 4 (the Schur complement lemma $[9,12]$ ). Given matrices $\mathbb{Q}=\mathfrak{Q}^{T}, \mathscr{R}=\mathscr{R}^{T}$, and $\mathcal{S}$ with appropriate dimensions, the following propositions are equivalent:

$$
\begin{array}{r}
{\left[\begin{array}{cc}
\mathscr{Q} & \mathcal{S} \\
(\star) & \mathscr{R}
\end{array}\right]>0,} \\
\left\{\begin{array}{l}
\mathscr{R}>0, \\
\mathbb{Q}-\mathcal{S}^{-1} \mathcal{S}^{\mathrm{T}}>0 .
\end{array}\right.
\end{array}
$$

Lemma 5 (the S-procedure lemma $[9,12])$. Let $\mathscr{F}_{0}, \ldots, \mathscr{F}_{p}$ $\in \mathbb{R}^{n \times n}$ be symmetric matrices. We consider the following conditions on $\mathscr{F}_{0}, \ldots, \mathscr{F}_{p}$ :

$$
\begin{aligned}
& \zeta^{\mathrm{T}} \mathscr{F}_{0} \zeta>0, \quad \forall \zeta \neq 0, \\
\text { s.t. } & \zeta^{\mathrm{T}} \mathscr{F}_{i} \zeta \geq 0, \quad \forall i=1, \ldots, p .
\end{aligned}
$$

If there exist scalar variables $\tau_{1} \geq 0, \ldots, \tau_{p} \geq 0$, such that

$$
\mathscr{F}_{0}-\sum_{i=1}^{p} \tau_{i} \mathscr{F}_{i}>0
$$

then (5) holds.

2.2. Class of Nonlinear Systems. A general class of continuous-time nonlinear systems with a control input $\mathbf{u}$ and under disturbance signal $\mathbf{w}$ is defined by the following form:

$$
\dot{\mathbf{x}}=\mathbf{F}(\mathbf{x}, \mathbf{u}, \mathbf{w}),
$$

where $\mathbf{x} \in \mathbb{R}^{n_{a}}, \mathbf{u} \in \mathbb{R}^{n_{u}}$, and $\mathbf{w} \in \mathbb{R}^{n_{w}}$.

In this work, we will consider a particular class of these nonlinear systems, for which the mathematical model is given by the following differential equation:

$$
\dot{\mathbf{x}}=\mathbf{A} \mathbf{x}+\mathbf{B} \mathbf{u}+\sum_{i=1}^{n_{f}} \mathbf{C}_{i} f_{i}(\mathbf{x})+\mathbf{E w},
$$

where $\mathbf{A} \in \mathbb{R}^{n_{x} \times n_{x}}, \mathbf{B} \in \mathbb{R}^{n_{x} \times n_{u}}, \mathbf{C}_{i} \in \mathbb{R}^{n_{x} \times 1}, \mathbf{E} \in \mathbb{R}^{n_{x} \times n_{w}}$, and the nonlinear functions $f_{i}(\mathbf{x})$ are scalars, i.e., $f_{i}(\mathbf{x}) \in \mathbb{R}$ for all $i=1, \ldots, n_{f}$. Moreover, we will consider that, in the nonlinear system ( 7$)$, we have $\mathbf{F}(0,0,0) \neq 0$. This means that the state $\mathbf{x}=0$ is not the equilibrium point. Hence, in the dynamics (8), we have 


$$
\sum_{i=1}^{n_{f}} \mathbf{C}_{i} f_{i}(0) \neq 0
$$

In addition, we will consider, without loss of generality, that

$$
\left|f_{i}(\mathbf{x})\right| \leq 1, \quad \forall i=1, \ldots, n_{f}
$$

Remark 1. In this work, we consider a class of nonlinear systems (8), in which the nonlinear terms $\mathbf{f}_{i}(\mathbf{x})$, for all $i=1, \ldots, n_{f}$, satisfy the constraints in (10). Our LMI-based approach for the design of a stabilizing control law $\mathbf{u}$, which will be designed in the sequel, can be applied for a general class of fully nonlinear systems such as (hyper) chaotic systems as in $[59,60]$, robotic/mechatronic systems as in $[43,46]$, and underactuated mechanical systems [61, 62], just to mention a few. Thus, the nonlinearity term, saying $\mathbf{h}(\mathbf{x})=\sum_{i=1}^{n_{f}} \mathbf{C}_{i} f_{i}(\mathbf{x})$, can be assumed to satisfy the following quadratic inequality [12]:

$$
\mathbf{h}^{\mathrm{T}}(\mathbf{x}) \mathbf{h}(\mathbf{x}) \leq \lambda^{2} \mathbf{x}^{\mathrm{T}} \mathbf{H}^{T} \mathbf{H} \mathbf{x},
$$

where $\lambda$ is a positive scalar and $\mathbf{H}$ is a constant matrix having appropriate dimension.

Some other research papers have considered ellipsoidal condition or one-sided Lipschitz condition on the nonlinearity $\mathbf{h}(\mathbf{x})$. For this subject, we refer the readers to, for example, $[3,8,60,63-65]$ and references therein.

2.3. Motivation Application: The Simplified Pitch Dynamics of the Helicopter Model. As an illustrative motivation application, we will consider in this work a simplified helicopter pitch dynamics model. The schematic model of the helicopter is shown in Figure 1. We refer our readers to [55-57] for further details on this simplified model. The pitch dynamics of the simplified helicopter model has two degrees of freedom with only one actuator. Such model was employed to develop new control strategies for mechatronic systems [55-57].

The nonlinear model of the pitch dynamics of the simplified helicopter is described by the following differential equations [55-57]:

$$
\begin{aligned}
\dot{x}_{1}= & x_{2}, \\
\dot{x}_{2}= & \frac{1}{I_{y y}}\left(-m_{\text {heli }} l_{c g x} \cos \left(x_{1}\right)-m_{\text {heli }} l_{c g z} g \sin \left(x_{1}\right)-F_{v M} x_{2}+u\right) \\
& +\frac{s}{I_{y y}} w,
\end{aligned}
$$

where $x_{1}$ and $x_{2}$ represent the pitch angle ( $\theta$ in Figure 1$)$ and pitch rate, respectively; $I_{y y}$ is the second moment around the $y$-axis; $m_{\text {heli }}$ is the mass of the helicopter; $l_{c g x}$ and $l_{c g z}$ are displacements from the center of mass (GC in Figure 1) relative to the rotation joint $B$ shown in Figure $1 ; F_{v M}$ is the pitch damping; $s$ is the variance of the moment disturbance; $u$ is the control torque exerted by the main blade of the helicopter around the $y$-axis; and $w$ is the external disturbance, which is modeled as an additive white noise representing the turbulent moments on the helicopter [55-57].

For simplicity, let us introduce the following change of variables: $a=-\left(F_{v M} / I_{y y}\right), b=1 / I_{y y}, c=-\left(m_{\text {heli }} l_{c g x} / I_{y y}\right)$, $d=-\left(m_{\text {heli }} l_{c g z} g / I_{y y}\right)$, and $e=s / I_{y y}$. Then, the nonlinear differential equations in (12) can be rewritten under the particular class (8), where $\mathbf{x}=\left[\begin{array}{l}x_{1} \\ x_{2}\end{array}\right], \mathbf{A}=\left[\begin{array}{ll}0 & 1 \\ 0 & a\end{array}\right], \mathbf{B}=\left[\begin{array}{l}0 \\ b\end{array}\right]$, $\mathbf{C}_{1}=\left[\begin{array}{l}0 \\ c\end{array}\right], \mathbf{C}_{2}=\left[\begin{array}{l}0 \\ d\end{array}\right], \mathbf{E}=\left[\begin{array}{l}0 \\ e\end{array}\right], f_{1}(\mathbf{x})=f_{1}\left(x_{1}\right)=\cos \left(x_{1}\right)$, and $f_{2}(\mathbf{x})=f_{2}\left(x_{1}\right)=\sin \left(x_{1}\right)$.

Notice that the two nonlinear terms $f_{1}(\mathbf{x})$ and $f_{2}(\mathbf{x})$ satisfy condition (10) and also constraint (9). Hence, the zero state, $\mathbf{x}=0$, is not the equilibrium (singular) point of the pitch dynamics of the helicopter model and then of the undisturbed uncontrolled nonlinear system (8), i.e., for $w=$ 0 and $u=0$.

Remark 2. It is worth to note that the model of the simple pitch dynamics (8) of the helicopter in Figure 1 was adopted from [55-57]. It is a simple model where the propeller dynamics (including motor, propeller interaction, air stream interaction, gyroscopic moments, and propeller flexing), the perturbation modelling (including wind gust modelling and payload modelling), and the cyclic/collective mechanism modelling are not considered in this study. Only the turbulent moments are taken into account as an external disturbance input, $w$. We emphasize that all these previous unmodelled dynamics can be lumped into a single term, saying that $\boldsymbol{\delta}(\mathbf{x})$ can be majored according to condition (11).

For more general and basic pitch models for helicopters that reproduce basic dynamic properties of these vehicles, we can refer to [66] for typical helicopter models or [67] for more modern applications.

2.4. Problem Statement. In the sequel, and for simplicity in developing and designing LMI stability conditions, we will consider that, in (8), $n_{f}=2$, and then the class of nonlinear systems to consider is as follows:

$$
\dot{\mathbf{x}}=\mathbf{A x}+\mathbf{B u}+\mathbf{C} f(\mathbf{x})+\mathbf{D} g(\mathbf{x})+\mathbf{E w}
$$

where $f(\mathbf{x})=f_{1}(\mathbf{x})$ and $g(\mathbf{x})=f_{2}(\mathbf{x})$ are the two scalar nonlinearities satisfying conditions (9) and (10).

As noted before, the zero state $\mathbf{x}=0$ is not the equilibrium point of the undisturbed uncontrolled nonlinear system (8) and then the simplified nonlinear system (13). Thus, we obtain $\mathbf{C} f(0)+\mathbf{D} g(0) \neq 0$.

Our objective in this work is to control dynamics (13) to the zero state by designing a feedback controller, even if the dynamics is under parametric uncertainties or subject to the disturbance vector $\mathbf{w}$. Thus, we assume that the disturbance signal $\mathbf{w}$ is bounded such that

$$
\mathbf{w}^{\mathrm{T}} \mathbf{w} \leq \rho,
$$

for some positive scalar $\rho$. 


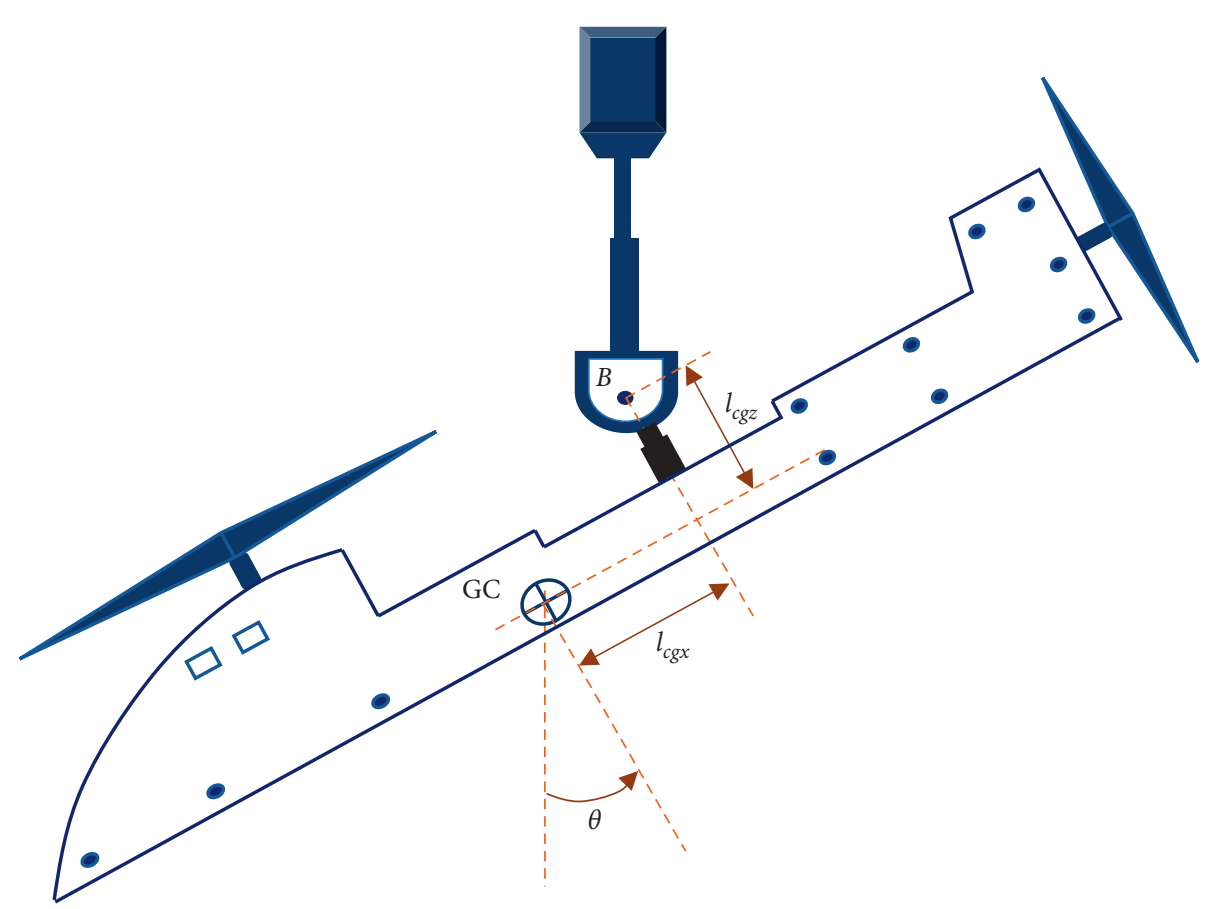

FIGURE 1: Simplified pitch model of the helicopter, from [55-57].

Furthermore, we assume that all parameters in the nonlinear system (13) are uncertain and are with a bounded norm. The values of these parameters presented in the nonlinear dynamics (13) are considered to be the nominal ones. Thus, the nonlinear system (13) will be considered as the nominal system, for which $(\mathbf{A}, \mathbf{B})$ is controllable.

Hence, under parametric uncertainties, the nominal system (13) will be rewritten as follows:

$$
\begin{aligned}
\dot{\mathbf{x}}= & (\mathrm{A}+\Delta \mathrm{A}) \mathbf{x}+(\mathbf{B}+\Delta \mathbf{B}) \mathbf{u}+(\mathbf{C}+\Delta \mathbf{C}) \mathbf{f}(\mathbf{x}) \\
& +(\mathbf{D}+\Delta \mathrm{D}) \mathbf{g}(\mathbf{x})+(\mathbf{E}+\Delta \mathrm{E}) \mathbf{w},
\end{aligned}
$$

where $\Delta \mathrm{A}, \Delta \mathrm{B}, \Delta \mathrm{C}, \Delta \mathrm{D}$, and $\Delta \mathrm{E}$ are matrices containing parametric uncertainties and satisfying the following expressions:

$$
\begin{aligned}
& \Delta \mathbf{A}=\sum_{i_{1}=1}^{q_{1}} \delta_{a}^{i_{1}} \mathbf{A}_{1}^{i_{1}} \mathbf{A}_{2}^{i_{1}}, \\
& \mathbf{\Delta} \mathbf{B}=\sum_{i_{2}=1}^{q_{2}} \delta_{b}^{i_{2}} \mathbf{B}_{1}^{i_{2}} \mathbf{B}_{2}^{i_{2}}, \\
& \mathbf{\Delta} \mathbf{C}=\sum_{i_{3}=1}^{q_{3}} \delta_{c}^{i_{3}} \mathbf{C}_{1}^{i_{3}} \mathbf{C}_{2}^{i_{3}}, \\
& \mathbf{\Delta} \mathbf{D}=\sum_{i_{4}=1}^{q_{4}} \delta_{d}^{i_{4}} \mathbf{D}_{1}^{i_{4}} \mathbf{D}_{2}^{i_{4}}, \\
& \mathbf{\Delta} \mathbf{E}=\sum_{i_{5}=1}^{q_{5}} \delta_{e}^{i_{5}} \mathbf{E}_{1}^{i_{5}} \mathbf{E}_{2}^{i_{5}},
\end{aligned}
$$

where the matrices $\mathbf{A}_{1}^{i_{1}}, \mathbf{A}_{2}^{i_{1}}, \mathbf{B}_{1}^{i_{2}}, \mathbf{B}_{2}^{i_{2}}, \mathbf{C}_{1}^{i_{3}}, \mathbf{C}_{2}^{i_{3}}, \mathbf{D}_{1}^{i_{4}}, \mathbf{D}_{2}^{i_{4}}, \mathbf{E}_{1}^{i_{5}}$, and $\mathbf{E}_{2}^{i_{5}}$ are with appropriate dimensions. Moreover, all the uncertainties $\delta_{a}^{i_{1}}, \delta_{b}^{i_{2}}, \delta_{c}^{i_{3}}, \delta_{d}^{i_{4}}$, and $\delta_{e}^{i_{5}}$, for all $i_{1}=1, \ldots, q_{1}$, $i_{2}=1, \ldots, q_{2}, \quad i_{3}=1, \ldots, q_{3}, \quad i_{4}=1, \ldots, q_{4}, \quad$ and $i_{5}=1, \ldots, q_{5}$, are with a bounded norm, i.e.,

$$
\left|\delta_{i}^{j}\right| \leq \bar{\delta}_{i}^{j}
$$

for all $i \in\{a, b, c, d, e\}$ and for all $j \in\left\{i_{1}, i_{2}, i_{3}, i_{4}, i_{5}\right\}$.

In addition, we will consider that the uncertain nonlinear dynamics (15) is subject to an input saturation of the controller $\mathbf{u}$ :

$$
-u_{\max }^{i} \leq u^{i} \leq u_{\max }^{i}, \quad \forall i=1, \ldots, n_{u},
$$

where $u_{\max }^{i}$, for all $i=1, \ldots, n_{u}$, are prescribed positive scalars and $u^{i}$ is the $i$ th element of the control vector $\mathbf{u}$.

The problem we are addressing in this work is to find, for the uncertain disturbed nonlinear system (15), an affine state-feedback control law

$$
\mathbf{u}=\mathbf{K x}+\mathbf{m},
$$

where $\mathbf{K} \in \mathbb{R}^{n_{u} \times n_{x}}$ and $\mathbf{m} \in \mathbb{R}^{n_{u}}$ are the feedback gains to design, for which the control law $\mathbf{u}$ is constrained according to condition (18). The proposed control law (19) is actually a linear state-feedback law augmented with the constant term, $\mathbf{m}$, to keep the system state around an operating point, that is, the zero-equilibrium point $\mathbf{x}=0$.

Let us define the following set:

$$
\mathscr{L}\left(\mathbf{K}, \mathbf{m}, \mathbf{u}_{\max }\right)=\left\{\mathbf{x} \in \mathbb{R}^{n_{x}}:|\mathbf{K} \mathbf{x}+\mathbf{m}| \leq \mathbf{u}_{\max }\right\},
$$

as the region in the state space where the feedback control law $\mathbf{m}$ in (19) is linear (affine) in terms of the state vector $\mathbf{x}$. 
In addition, let $\mathbf{P} \in \mathbb{R}^{n_{x} \times n_{x}}$ be a positive-definite symmetric matrix and denote the Lyapunov level set as

$$
\varepsilon(\mathbf{P}, \gamma)=\left\{\mathbf{x} \in \mathbb{R}^{n_{x}}: x^{\mathrm{T}} \mathbf{P} \mathbf{x} \leq \gamma\right\}
$$

where $\gamma$ is a prescribed positive scalar.

The level set $\varepsilon(\mathbf{P}, \gamma)$ defined by (21) is an invariant set $[5,52]$. Moreover, it is associated with the Lyapunov function $V(\mathbf{x})=\mathbf{x}^{\mathrm{T}} \mathbf{P} \mathbf{x}$ for the closed-loop system. Consequently, $\varepsilon(\mathbf{P}, \gamma)$ is an invariant set of the nonlinear system (15) with the control law (19) in the sense of the following definition.

Definition 1. A set $\mathcal{S} \subset \mathbb{R}^{n_{x}}$ is said to be invariant with respect to motion of system (14), if for all initial conditions $\mathbf{x}(0) \in \mathcal{S}$, the motion of the system $\mathbf{x}(t)$ remains in $\mathcal{S}$ for all $t>0$.

Accordingly, the ellipsoid $\varepsilon(\mathbf{P}, \gamma)$ is said to be contractively invariant if $\dot{V}(\mathbf{x})<0$ for all $\mathbf{x} \in \mathcal{E}(\mathbf{P}, \gamma) \backslash\{0\}$. Clearly, if $\varepsilon(\mathbf{P}, \gamma)$ is contractively invariant, then it is inside the domain of attraction $[5,52]$.

According to Lemma 1 and by posing $\eta=\mu \gamma$, we will have

$$
\dot{V}(\mathbf{x}) \leq-\mu(V(\mathbf{x})-\gamma)
$$

Thus, on the boundary of the set $\varepsilon(\mathbf{P}, \gamma)$, we have $\mathbf{x}^{\mathrm{T}} \mathbf{P} \mathbf{x}=\gamma$. Hence, $\dot{V}(\mathbf{x})<0$. It follows that $\varepsilon(\mathbf{P}, \gamma)$ is a strictly ellipsoidal invariant set $[5,47]$.

Condition (22) will be used in the sequel in order to design the robust stabilization conditions of the closed-loop nonlinear system (15) with the norm-bounded parametric uncertainties (17) under the bounded external disturbance (14) and subject to the saturation (18) of the affine statefeedback control law (19) under condition (35).

As we look for stability conditions for the uncertain disturbed nonlinear system (15) under the saturated feedback control law $u$ inside the invariant ellipsoid (21), we should have the following constraint:

$$
\varepsilon(\mathbf{P}, \gamma) \subset \mathscr{L}\left(\mathbf{K}, \mathbf{m}, \mathbf{u}_{\max }\right) .
$$

Therefore, if for a certain Lyapunov matrix $\mathbf{P}=\mathbf{P}^{\mathrm{T}}>0$ satisfying conditions (22) and (23), then taking any initial condition in the invariant ellipsoid $\varepsilon(\mathbf{P}, \gamma)$, we will have $u=$ $\mathbf{K} \mathbf{x}+\mathbf{m}$ belongs to the set $\mathscr{L}\left(\mathbf{K}, \mathbf{m}, \mathbf{u}_{\max }\right)$.

In the sequel, we will develop conditions under which the ellipsoid $\varepsilon(\mathbf{P}, \gamma)$ is contractively invariant and the disturbed uncertain nonlinear system (15) is robustly stable by means of the saturated affine state-feedback control law (19). Moreover, we will show how to compute the gains $\mathbf{K}$ and $\mathbf{m}$ and the Lyapunov matrix $\mathbf{P}$, which determine the largest ellipsoid $\varepsilon(\mathbf{P}, \gamma)$ and hence obtain an estimate of the domain of attraction.

It is worth noting that, for the case of the pitch dynamics of the helicopter model, the uncertainty matrices are $\Delta \mathbf{A}=\left[\begin{array}{cc}0 & 0 \\ 0 & \delta_{a}\end{array}\right], \Delta \mathbf{B}=\left[\begin{array}{c}0 \\ \delta_{b}\end{array}\right], \Delta \mathbf{C}=\left[\begin{array}{c}0 \\ \delta_{c}\end{array}\right], \Delta \mathbf{D}=\left[\begin{array}{c}0 \\ \delta_{d}\end{array}\right]$, and $\Delta \mathbf{E}=\left[\begin{array}{c}0 \\ \delta_{e}\end{array}\right]$.
Moreover, it is easy to show that these uncertainty matrices $\Delta \mathrm{A}, \Delta \mathbf{B}, \Delta \mathrm{C}, \Delta \mathrm{D}$, and $\Delta \mathrm{E}$ can be rewritten like so:

$$
\begin{aligned}
\Delta \mathbf{A} & =\delta_{a} \mathbf{F F}^{\mathrm{T}}, \\
\Delta \mathbf{B} & =\delta_{b} \mathbf{F}, \\
\Delta \mathbf{C} & =\delta_{c} \mathbf{F}, \\
\Delta \mathbf{D} & =\delta_{d} \mathbf{F} \\
\Delta \mathbf{E} & =\delta_{e} \mathbf{F},
\end{aligned}
$$

where $\mathbf{F}=\left[\begin{array}{ll}0 & 1\end{array}\right]^{\mathrm{T}}$.

Thus, in (16a)-(16e), we have $q_{1}=1, q_{2}=1, q_{3}=1$, $q_{4}=1$, and $q_{5}=1$ and then $\mathbf{A}_{1}^{1}=\mathbf{F}, \mathbf{A}_{2}^{1}=\mathbf{F}^{\mathrm{T}}, \mathbf{B}_{1}^{1}=\mathbf{F}, \mathbf{B}_{2}^{1}=1$, $\mathbf{C}_{1}^{1}=\mathbf{F}, \mathbf{C}_{2}^{1}=1, \mathbf{D}_{1}^{1}=\mathbf{F}, \mathbf{D}_{2}^{1}=1, \mathbf{E}_{1}^{1}=\mathbf{F}$, and $\mathbf{E}_{2}^{1}=1$.

Furthermore, the uncertainties $\delta_{a}, \delta_{b}, \delta_{c}, \delta_{d}$, and $\delta_{e}$ should satisfy condition (17) and then $\left|\delta_{i}\right| \leq \bar{\delta}_{i}$, for all $i \in\{a, b, c, d, e\}$.

In the sequel of this work, without loss of generality, we will take in (16a)-(16e), $q_{1}=1, q_{2}=1, q_{3}=1, q_{4}=1$, and $q_{5}=1$. Moreover, we will consider a single control input $\mathbf{u}$ and also a single disturbance signal $\mathbf{w}$, i.e., $n_{u}=1$ and $n_{w}=1$. We will also consider the expression of the uncertainty matrices in (24).

\section{Design of the Robust Saturated Affine State- Feedback Controller}

In this section, we develop conditions satisfying robust stabilization of the uncertain disturbed nonlinear dynamics (15) under the affine state-feedback control law (19) subject to the saturation constraint (18). Thus, these stability conditions must verify constraints (22) and (23). We show first that the stability conditions are expressed in terms of BMIs. Next, by means of previously provided technical lemmas, we transform these BMIs into LMIs. Finally, we present an optimization problem providing the allowable maximum values of the parametric uncertainties $\delta_{a}, \delta_{b}, \delta_{c}$, $\delta_{d}$, and $\delta_{e}$.

3.1. BMI-Based Stability Conditions. These stability conditions of the disturbed uncertain nonlinear system (15) under the saturated affine state-feedback control law defined by expression (19), constraint (18), and condition (35) are presented in the following theorem.

Theorem 1. The nonlinear system (15) with normbounded parametric uncertainties (17), under the bounded external disturbance (14), is robustly stabilizable by implementing the affine state-feedback control law (19) subject to the saturation constraint (18), if for some fixed positive parameters $\gamma, \varepsilon_{m}, \bar{\delta}_{a}, \bar{\delta}_{b}, \bar{\delta}_{c}, \bar{\delta}_{d}$, and $\bar{\delta}_{e}$, there exist a symmetric matrix $\mathbf{P}>0$, a feedback matrix $\mathbf{K}$, a feedback constant $m$, and some positive scalars $\varepsilon_{1}, \varepsilon_{2}, \varepsilon_{3}, \varepsilon_{4}, \varepsilon_{5}, \varepsilon_{6}, \varepsilon_{7}$, $\varepsilon_{8}, \varepsilon_{9}, \mu$, and $\eta$ such that the following set of matrix inequalities is feasible: 


$$
\begin{array}{r}
{\left[\begin{array}{cc}
(\mathbf{P A}+\mathbf{P B K})+(*)+\mu \mathbf{P}+\mathbb{A}_{1} & \mathbf{P B} m \\
(\star) & -\mu \gamma+\xi
\end{array}\right]<0,} \\
{\left[\begin{array}{cc}
\mathbf{K}^{\mathrm{T}} \mathbf{K}-\eta \mathbf{P} & \mathbf{K}^{T} m \\
(\star) & \eta \gamma+m^{T} m-u_{\max }^{2}
\end{array}\right]<0,} \\
(\mathbf{B} m+\widehat{\mathbf{C}})^{\mathrm{T}}(\mathbf{B} m+\widehat{\mathbf{C}})<\varepsilon_{m},
\end{array}
$$

where

$$
\begin{aligned}
\mathbb{A}_{1}= & \varepsilon_{1} \mathbf{P C C}^{T} \mathbf{P}+\varepsilon_{2} \mathbf{P D D}^{T} \mathbf{P}+\varepsilon_{3} \mathbf{P E E}^{T} \mathbf{P} \\
& +\left(\varepsilon_{4}+\varepsilon_{5}+\varepsilon_{6}+\varepsilon_{7}+\varepsilon_{8}+\varepsilon_{9}\right) \mathbf{P F F}^{\mathrm{T}} \mathbf{P}+\varepsilon_{4}^{-1} \bar{\delta}_{a}^{2} \mathbf{F F}^{\mathrm{T}} \\
& +\varepsilon_{5}^{-1} \bar{\delta}_{b}^{2} \mathbf{K}^{\mathrm{T}} \mathbf{K}, \\
\xi= & \varepsilon_{1}^{-1}+\varepsilon_{2}^{-1}+\varepsilon_{3}^{-1}+\varepsilon_{6}^{-1} \bar{\delta}_{b}^{2} m^{\mathrm{T}} m+\varepsilon_{7}^{-1} \bar{\delta}_{c}^{2}+\varepsilon_{8}^{-1} \bar{\delta}_{d}^{2}+\varepsilon_{9}^{-1} \bar{\delta}_{e}^{2} \rho^{2} .
\end{aligned}
$$

Proof. By using system (15) and control law (19), the derivative of the Lyapunov function, $V(\mathbf{x})=\mathbf{x}^{\mathrm{T}} \mathbf{P} \mathbf{x}$, is given by

$$
\begin{aligned}
\dot{V}(\mathbf{x})= & 2 \mathbf{x}^{\mathrm{T}} \mathbf{P} \overline{\mathbf{A}} \mathbf{x}+2 \mathbf{x}^{\mathrm{T}} \mathbf{P B} m+2 \mathbf{x}^{\mathrm{T}} \mathbf{P C} \mathbf{f}(\mathbf{x})+2 \mathbf{x}^{\mathrm{T}} \mathbf{P D g}(\mathbf{x}) \\
& +2 \mathbf{x}^{\mathrm{T}} \mathbf{P} \mathbf{E} w+2 \mathbf{x}^{\mathrm{T}} \mathbf{P} \boldsymbol{\Delta} \mathbf{A} \mathbf{x}+2 \mathbf{x}^{\mathrm{T}} \mathbf{P} \boldsymbol{\Delta} \mathbf{B K} \mathbf{x}+2 \mathbf{x}^{\mathrm{T}} \mathbf{P} \boldsymbol{\Delta} \mathbf{B} m \\
& +2 \mathbf{x}^{\mathrm{T}} \mathbf{P} \boldsymbol{\Delta} \mathbf{C} \mathbf{f}(\mathbf{x})+2 \mathbf{x}^{\mathrm{T}} \mathbf{P} \boldsymbol{\Delta} \mathbf{D} \mathbf{g}(x)+2 \mathbf{x}^{\mathrm{T}} \mathbf{P} \boldsymbol{\Delta} \mathbf{E} w,
\end{aligned}
$$

with $\overline{\mathbf{A}}=\mathbf{A}+\mathbf{B K}$.

We now consider condition (22) on the Lyapunov function $V(\mathbf{x})$ and also conditions (10), (14), and (17). Then, using expressions of the uncertainties matrices in (24) and relying on the Young relation (Lemma 2), we obtain the following condition:

$$
\begin{aligned}
\dot{V}(\mathbf{x})+\mu(V(\mathbf{x})-\gamma) \leq & \mu \mathbf{x}^{\mathrm{T}} \mathbf{P} \mathbf{x}-\mu \gamma+2 \mathbf{x}^{\mathrm{T}} \mathbf{P} \overline{\mathbf{A}} \mathbf{x}+2 \mathbf{x}^{\mathrm{T}} \mathbf{P B} m \\
& +\varepsilon_{1} x^{\mathrm{T}} \mathbf{P} \mathbf{P C}^{\mathrm{T}} \mathbf{P} \mathbf{x}+\varepsilon_{1}^{-1}+\varepsilon_{2}^{-1}+\varepsilon_{3}^{-1} \\
& +\varepsilon_{2} \mathbf{x}^{\mathrm{T}} \mathbf{P} \mathbf{P D}^{\mathrm{T}} \mathbf{P} \mathbf{x} \\
& +\varepsilon_{3} \mathbf{x}^{\mathrm{T}} \mathbf{P} \mathbf{P E}^{\mathrm{T}} \mathbf{P} \mathbf{x}+\left(\varepsilon_{4}+\varepsilon_{5}+\varepsilon_{6}+\varepsilon_{7}\right. \\
& \left.+\varepsilon_{8}+\varepsilon_{9}\right) \mathbf{x}^{\mathrm{T}} \mathbf{P} \mathbf{F} F^{\mathrm{T}} \mathbf{P} \mathbf{x} \\
& +\varepsilon_{4}^{-1} \bar{\delta}_{a}^{2} x^{\mathrm{T}} \mathbf{F} \mathbf{F}^{\mathrm{T}} \mathbf{x}+\varepsilon_{5}^{-1} \bar{\delta}_{b}^{2} \mathbf{x}^{\mathrm{T}} \mathbf{K}^{\mathrm{T}} \mathbf{K} \mathbf{x} \\
& +\varepsilon_{6}^{-1} \bar{\delta}_{b}^{2} m^{\mathrm{T}} m+\varepsilon_{7}^{-1} \bar{\delta}_{c}^{2} \\
& +\varepsilon_{8}^{-1} \bar{\delta}_{d}^{2}+\varepsilon_{9}^{-1} \bar{\delta}_{e}^{2} \rho^{2}<0 .
\end{aligned}
$$

This last inequality constraint can be rewritten as follows:

$$
\left[\begin{array}{l}
\mathbf{x} \\
1
\end{array}\right]^{\mathrm{T}}\left[\begin{array}{cc}
\mathbf{A}_{2}+\mu \mathbf{P} & \mathbf{P B} m \\
(\star) & -\mu \gamma+\xi
\end{array}\right]\left[\begin{array}{l}
\mathbf{x} \\
1
\end{array}\right]<0,
$$

where $\quad \mathbb{A}_{2}=\mathbf{P} \overline{\mathbf{A}}+(\star)+\varepsilon_{1} \mathbf{P C C} \mathbf{C}^{\mathrm{T}} \mathbf{P}+\varepsilon_{2} \mathbf{P} \mathbf{D D}^{\mathrm{T}} \mathbf{P}+\varepsilon_{3} \mathbf{P E E}^{\mathrm{T}} \mathbf{P}$ $+\left(\varepsilon_{4}+\varepsilon_{5}+\varepsilon_{6}+\varepsilon_{7}+\varepsilon_{8}+\varepsilon_{9}\right) \mathbf{P F F} \mathbf{F}^{\mathrm{T}} \mathbf{P}+\varepsilon_{4}^{-1} \bar{\delta}_{a}^{2} \mathbf{F F}^{\mathrm{T}}+\varepsilon_{5}^{-1} \bar{\delta}_{b}^{2} \mathbf{K}^{\mathrm{T}} \mathbf{K}$ and $\quad \xi=\varepsilon_{1}^{-1}+\varepsilon_{2}^{-1}+\varepsilon_{3}^{-1}+\varepsilon_{6}^{-1} \bar{\delta}_{b}^{2} m^{T} m+\varepsilon_{7}^{-1} \bar{\delta}_{c}^{2}+\varepsilon_{8}^{-1} \bar{\delta}_{d}^{2}+$ $\varepsilon_{9}^{-1} \bar{\delta}_{e}^{2} \rho^{2}$.

Hence, we obtain the matrix inequality (25).
Furthermore, from expressions (20) and (21), rewrite $\mathbf{x} \in \varepsilon(\mathbf{P}, \gamma)$ as

$$
\left[\begin{array}{l}
\mathbf{x} \\
1
\end{array}\right]^{\mathrm{T}}\left[\begin{array}{cc}
\mathbf{P} & \mathcal{O} \\
(\star) & -\gamma
\end{array}\right]\left[\begin{array}{l}
\mathbf{x} \\
1
\end{array}\right] \leq 0
$$

and $\mathbf{x} \in \mathscr{L}\left(\mathbf{K}, \mathbf{m}, \mathbf{u}_{\max }\right)$ as

$$
\left[\begin{array}{c}
\mathbf{x} \\
1
\end{array}\right]^{\mathrm{T}}\left[\begin{array}{cc}
\mathbf{K}^{\mathrm{T}} \mathbf{K} & \mathbf{K}^{\mathrm{T}} m \\
(\star) & m^{T} m-u_{\max }^{2}
\end{array}\right]\left[\begin{array}{l}
\mathbf{x} \\
1
\end{array}\right] \leq 0
$$

It is worth to note that the condition $\varepsilon(\mathbf{P}, \gamma) \subset \mathscr{L}(\mathbf{K}, \mathbf{m}$, $\mathbf{u}_{\max }$ ) (constraint (23)) is nothing than the implication (32) $\Longrightarrow$ (33). Therefore, by using the S-procedure lemma, this implication condition is equivalent to the existence of a positive scalar $\eta$ such that the matrix inequality (26) holds.

As noted in the beginning of the previous section, the zero state $\mathbf{x}=0$ is not the equilibrium point. Then, under the affine state-feedback control law (19) and for a certain and undisturbed dynamics (that is, for the nominal dynamics (13)), the zero-equilibrium point should verify the following condition:

$$
\mathbf{B} m+\mathbf{C} f(0)+\mathbf{D} g(0)=0 \text {. }
$$

Posing $\widehat{\mathbf{C}}=\mathbf{C} f(0)+\mathbf{D} g(0)$, then relation (34) is recast as follows:

$$
\mathbf{B} m+\widehat{\mathbf{C}}=0 .
$$

It is worth to note that, in condition (35), the term $\mathbf{B} m+$ $\widehat{\mathbf{C}}$ is a column vector. Then, such relation in (35) can be written in a (scalar) matrix form like so:

$$
(\mathbf{B} m+\widehat{\mathbf{C}})^{\mathrm{T}}(\mathbf{B} m+\widehat{\mathbf{C}})=0 .
$$

For a small enough positive scalar $\varepsilon_{m}$, equality (36) can be transformed into condition (27). This completes the proof of Theorem 1.

It is worth mentioning that the three matrix inequalities (25)-(27) in Theorem 1 are BMIs, which are hardly tractable numerically. Thus, our objective is to convert these BMIs (25)-(27) into LMIs, which is the objective of the next section.

Remark 3. Recall that, in the previous development of the BMI stability conditions, we have considered a single control input $u$ and then $n_{u}=1$. Hence, we used one row matrix gain $\mathbf{K}$ and one scalar gain $m$. This choice has led to the design of only one condition (26) satisfying the saturation condition of the controller $u$. For a general case and then for $n_{u}>1, \mathbf{K} \in \mathbb{R}^{n_{u} \times n_{x}}$, $\mathbf{m} \in \mathbb{R}^{n_{u}}$, and $\mathbf{u}_{\max } \in \mathbb{R}^{n_{u}}$, the stability condition (26) becomes

$$
\begin{aligned}
& {\left[\begin{array}{cc}
\mathbf{K}^{\mathrm{T}} \mathbf{e}_{i}^{\mathrm{T}} \mathbf{e}_{i} \mathbf{K}-\eta_{i} \mathbf{P} & \mathbf{K}^{\mathrm{T}} \mathbf{e}_{i}^{\mathrm{T}} \mathbf{e}_{i} \mathbf{m} \\
(\star) & \eta_{i} \gamma+\mathbf{m}^{\mathrm{T}} \mathbf{e}_{i}^{\mathrm{T}} \mathbf{e}_{i} \mathbf{m}-\mathbf{u}_{\max }^{\mathrm{T}} \mathbf{e}_{i}^{\mathrm{T}} \mathbf{e}_{i} \mathbf{u}_{\max }
\end{array}\right]<0,} \\
& \forall i=1, \ldots, n_{u} \text {, }
\end{aligned}
$$

where $\mathbf{e}_{i}=\underbrace{(0, \ldots, 0, \overbrace{1}^{i^{\text {th }}}, 0, \ldots, 0)} \in \mathbb{R}^{1 \times n_{u}}$ and $\eta_{i}>0$ for all $i=1, \ldots, n_{u}$. 
3.2. LMI-Based Stability Conditions. In this part, we aim at linearizing the three BMIs (25)-(27) in Theorem 1. Thus, we first linearize the BMI (25). After that, we linearize the BMI (26) and the BMI (27).

3.2.1. Linearization of the BMI (25). Let us put first $\mathbf{S}=\mathbf{P}^{-1}$. We premultiply and postmultiply the BMI (25) by the matrix $\operatorname{diag}(\mathbf{S}, 1)$. Then, we obtain

$$
\left[\begin{array}{cc}
(\mathbf{A S}+\mathbf{B R})+(\star)+\mu \mathbf{S}+\mathbb{A}_{3} & \mathbf{B} m \\
(*) & -\mu \gamma+\xi
\end{array}\right]<0,
$$

where $\mathbf{R}=\mathbf{K S}$ and $\mathbb{A}_{3}=\varepsilon_{1} \mathbf{C C}^{\mathrm{T}}+\varepsilon_{2} \mathbf{D D}^{\mathrm{T}}+\varepsilon_{3} \mathbf{E E}^{\mathrm{T}}+\left(\varepsilon_{4}+\right.$ $\left.\varepsilon_{5}+\varepsilon_{6}+\varepsilon_{7}+\varepsilon_{8}+\varepsilon_{9}\right) \mathbf{F F}^{\mathrm{T}}+\varepsilon_{4}^{-1} \bar{\delta}_{a}^{2} \mathbf{S} \mathbf{F F}^{\mathrm{T}} \mathbf{S}+\varepsilon_{5}^{-1} \bar{\delta}_{b}^{2} \mathbf{R}^{\mathrm{T}} \mathbf{R}$.

Based on the Schur complement lemma, matrix inequality (38) is equivalent to

$$
\left[\begin{array}{cccc}
\mathbb{A}_{4}+\mu \mathbf{S} & \mathbf{B} m & \mathbb{A}_{5} & \mathcal{O} \\
(\star) & -\mu \gamma & \mathcal{O} & \mathbb{A}_{7} \\
(\star) & (\star) & -\mathbb{A}_{6} & \mathcal{O} \\
(\star) & (\star) & (\star) & -\mathbb{A}_{8}
\end{array}\right]<0
$$

where $\mathbb{A}_{4}=(\mathbf{A S}+\mathbf{B R})+(\star)+\varepsilon_{1} \mathbf{C C}^{\mathrm{T}}+\varepsilon_{2} \mathbf{D D}^{\mathrm{T}}+\varepsilon_{3} \mathbf{E E}^{\mathrm{T}}+$ $\left(\varepsilon_{4}+\varepsilon_{5}+\varepsilon_{6}+\varepsilon_{7}+\varepsilon_{8}+\varepsilon_{9}\right) \mathbf{F F}^{\mathrm{T}}, \quad \mathrm{A}_{5}=\left[\bar{\delta}_{a} \mathbf{S F} \bar{\delta}_{b} \mathbf{R}^{T}\right], \quad \mathbb{A}_{6}=$ $\operatorname{diag}\left(\varepsilon_{4}, \varepsilon_{5}\right), \quad \mathbb{A}_{7}=\left[\begin{array}{lllllll}1 & 1 & 1 & \bar{\delta}_{b} m^{\mathrm{T}} \bar{\delta}_{c} \bar{\delta}_{d} \bar{\delta}_{e} \rho\end{array}\right]$, and $\mathbb{A}_{8}=$ $\operatorname{diag}\left(\varepsilon_{1}, \varepsilon_{2}, \varepsilon_{3}, \varepsilon_{6}, \varepsilon_{7}, \varepsilon_{8}, \varepsilon_{9}\right)$.

3.2.2. Linearization of the BMI (26). We premultiply and postmultiply the BMI (26) by the matrix $\operatorname{diag}(\mathbf{S}, 1)$. As a result, we obtain

$$
\left[\begin{array}{cc}
\mathbf{R}^{\mathrm{T}} \mathbf{R}-\eta \mathbf{S} & \mathbf{R}^{T} m \\
(\star) & -u_{\max }^{2}+m^{T} m+\eta \gamma
\end{array}\right]<0 .
$$

Let us introduce a new change of variables by posing $\mathbf{G}=\left[\begin{array}{ll}\mathbf{R}^{T} & \mathcal{O}\end{array}\right], \mathbf{L}=\left[\begin{array}{c}m \\ 1\end{array}\right], \mathbf{M}=\left[\begin{array}{cc}1 & 0 \\ 0 & 1 / \eta \gamma\end{array}\right]$, and $\alpha=-u_{\max }^{2}$. Then, we have $\mathbf{R}^{\mathrm{T}} \mathbf{R}=\mathbf{G G}^{\mathrm{T}}=\mathbf{G M G}^{\mathrm{T}}, \mathbf{R}^{\mathrm{T}} m=\mathbf{G L}$, and $-u_{\max }^{2}+m^{\mathrm{T}} m+\eta \gamma=\alpha+\mathbf{L}^{\mathrm{T}} \mathbf{M}^{-1} \mathbf{L}$. obtain

Then, by applying the Schur lemma to inequality (40), we

$$
\begin{array}{r}
-\eta \mathbf{S}+\mathbf{G} \mathbf{M G} \mathbf{G}^{\mathrm{T}}-\mathbf{G} \mathbf{L} \psi^{-1} \mathbf{L}^{\mathrm{T}} \mathbf{G}^{\mathrm{T}}<0, \\
\psi=\alpha+\mathbf{L}^{\mathrm{T}} \mathbf{M}^{-1} \mathbf{L}<0 .
\end{array}
$$

Relying on the matrix inversion lemma (see Lemma 3), its follows that

$$
\psi^{-1}=\alpha^{-1}-\alpha^{-2} \mathbf{L}^{T} \mathbf{H}^{-1} \mathbf{L},
$$

with

$$
\mathbf{H}=\mathbf{M}+\alpha^{-1} \mathbf{L L}^{\mathrm{T}} \text {. }
$$

Then, by substituting expression (43) in inequality (41), this last inequality (41) can be rewritten like so:

$$
-\eta \mathbf{S}+\mathbf{G} \mathbf{M G} \mathbf{G}^{\mathrm{T}}-\alpha^{-1} \mathbf{G} \mathbf{L} \mathbf{L}^{\mathrm{T}} \mathbf{G}^{\mathrm{T}}+\alpha^{-2} \mathbf{G} \mathbf{L} \mathbf{L}^{\mathrm{T}} \mathbf{H}^{-1} \mathbf{L L}^{\mathrm{T}} \mathbf{G}^{T}<0 .
$$
that

Through expression (44), it is straightforward to show

$$
\alpha^{-1} \mathbf{G L L}^{\mathrm{T}}=\mathbf{G H}-\mathbf{G M} .
$$

Therefore, based on this relation, we can note the following:

$$
\alpha^{-1} \mathbf{G} \mathbf{L L}^{\mathrm{T}} \mathbf{G}^{\mathrm{T}}=\mathbf{G H G}^{\mathrm{T}}-\mathbf{G M G}^{\mathrm{T}},
$$

$$
\alpha^{-2} \mathbf{G L L}^{\mathrm{T}} \mathbf{H}^{-1} \mathbf{L L}^{\mathrm{T}} \mathbf{G}^{\mathrm{T}}=\mathbf{G} \mathbf{H G}^{\mathrm{T}}-2 \mathbf{G M G}^{\mathrm{T}}+\mathbf{G M H}^{-1} \mathbf{M} \mathbf{G}^{\mathrm{T}}
$$

Thus, as $\mathbf{G M}=\mathbf{G}$, substituting relations (47) and (48) in inequality (45) yields

$$
\eta \mathbf{S}-\mathbf{G H}^{-1} \mathbf{G}^{\mathrm{T}}>0 .
$$

Accordingly, inequality (41) is transformed into inequality (49).

Our concern now is the auxiliary condition (42). As $\mathbf{M}>0$, then through the Schur lemma, inequality (42) is equivalent to

$$
\left[\begin{array}{cc}
\alpha & \mathbf{L}^{\mathrm{T}} \\
(\star) & -\mathbf{M}
\end{array}\right]<0 .
$$

By applying again the Schur lemma on inequality (50), we obtain

$$
-\mathbf{M}-\alpha^{-1} \mathbf{L L}^{\mathrm{T}}<0 .
$$

Hence, it follows that $\mathbf{H}>0$. Thus, by multiplying inequality (49) by $\left(\eta^{-1}\right)$ and relying on the Schur lemma, we obtain the following matrix inequality:

$$
\left[\begin{array}{cc}
\mathbf{S} & \mathbf{G} \\
(\star) & \eta \mathbf{H}
\end{array}\right]>0
$$

Since $\alpha=-u_{\max }^{2}$, then we can write the following:

$$
\eta \mathbf{H}=\eta \mathbf{M}-\frac{\eta}{u_{\max }^{2}} \mathbf{L L}^{\mathrm{T}}
$$

with $\eta \mathbf{M}=\left[\begin{array}{cc}\eta & 0 \\ 0 & 1 / \gamma\end{array}\right]$

Therefore, by taking into account relation (53), the Schur complement lemma states that inequality (52) is equivalent to

$$
\left[\begin{array}{ccc}
\mathbf{S} & \mathbf{G} & \boldsymbol{O} \\
(\star) & \eta \mathbf{M} & \mathbf{L} \\
(\star) & (\star) & \frac{u_{\max }^{2}}{\eta}
\end{array}\right]>0 .
$$

Hence, the BMI (26) is converted into the LMI (54), where the two parameters $\gamma$ and $\eta$ should be fixed a priori. 
3.2.3. Linearization of the BMI (27). We now consider the BMI (27). It can be rewritten like so:

$$
m^{\mathrm{T}} \mathbf{B}^{\mathrm{T}} \mathbf{B} m+2 \mathbf{C}^{\mathrm{T}} \mathbf{B} m+\widehat{\mathbf{C}}^{\mathrm{T}} \widehat{\mathbf{C}}<\varepsilon_{m}
$$

By applying the Schur lemma, this matrix inequality (55) is equivalent to the following LMI:

$$
\left[\begin{array}{cc}
\widehat{\mathbf{C}}^{\mathrm{T}} \widehat{\mathbf{C}}+2 \widehat{\mathbf{C}}^{\mathrm{T}} \mathbf{B} m-\varepsilon_{m} & m^{T} \mathbf{B}^{T} \\
(\star) & -\mathscr{I}
\end{array}\right]<0 .
$$

3.2.4. LMI Conditions. According to the previous linearization results, we state the following new theorem.

Theorem 2. Assume that, for some positive parameters $\gamma, \mu$, $\eta, \varepsilon_{m}, \rho, \bar{\delta}_{a}, \bar{\delta}_{b}, \bar{\delta}_{c}, \bar{\delta}_{d}$, and $\bar{\delta}_{e}$ fixed a priori, there exist $a$ symmetric matrix $\mathbf{S}$, a matrix $\mathbf{R}$, a scalar $m$, and some scalars $\varepsilon_{1}, \varepsilon_{2}, \varepsilon_{3}, \varepsilon_{4}, \varepsilon_{5}, \varepsilon_{6}, \varepsilon_{7}, \varepsilon_{8}$, and $\varepsilon_{9}$ so that the following set of LMIs is feasible:

$$
\begin{aligned}
& {\left[\begin{array}{cccc}
\mathbb{A}_{4}+\mu \mathbf{S} & \mathbf{B} m & \mathbb{A}_{5} & \mathscr{O} \\
(\star) & -\mu \gamma & \mathcal{O} & \mathbb{A}_{7} \\
(\star) & (\star) & -\mathbb{A}_{6} & \mathscr{O} \\
(\star) & (\star) & (\star) & -\mathbb{A}_{8}
\end{array}\right]<0,} \\
& {\left[\begin{array}{cccc}
\mathbf{S} & \mathbf{R}^{\mathrm{T}} & \mathcal{O} & \mathcal{O} \\
(\star) & \eta & \mathcal{O} & m \\
(\star) & (\star) & \frac{1}{\gamma} & 1 \\
(\star) & (\star) & (\star) & \frac{u_{\max }^{2}}{\eta}
\end{array}\right]>0,} \\
& {\left[\begin{array}{cc}
\widehat{\mathbf{C}}^{\mathrm{T}} \widehat{\mathbf{C}}+2 \widehat{\mathbf{C}}^{\mathrm{T}} \mathbf{B} m-\varepsilon_{m} & m^{T} \mathbf{B}^{T} \\
(\star) & -\mathscr{I}
\end{array}\right]<0,}
\end{aligned}
$$

where

$$
\begin{aligned}
& \mathbb{A}_{4}=(\mathbf{A S}+\mathbf{B R})+(\star)+\varepsilon_{1} \mathbf{C C}^{\mathrm{T}}+\varepsilon_{2} \mathbf{D D}^{\mathrm{T}}+\varepsilon_{3} \mathbf{E E}^{\mathrm{T}} \\
& +\left(\varepsilon_{4}+\varepsilon_{5}+\varepsilon_{6}+\varepsilon_{7}+\varepsilon_{8}+\varepsilon_{9}\right) \mathbf{F F}^{\mathrm{T}}, \\
& \mathbb{A}_{5}=\left[\begin{array}{lll}
\bar{\delta}_{a} & S F & \bar{\delta}_{b} \mathbf{R}^{\mathrm{T}}
\end{array}\right] \text {, } \\
& \mathbb{A}_{6}=\operatorname{diag}\left(\varepsilon_{4}, \varepsilon_{5}\right) \text {, } \\
& \mathbb{A}_{7}=\left[\begin{array}{llllllll}
1 & 1 & 1 & \bar{\delta}_{b} m^{\mathrm{T}} & \bar{\delta}_{c} & \bar{\delta}_{d} & \bar{\delta}_{e} \rho
\end{array}\right], \\
& \mathbb{A}_{8}=\operatorname{diag}\left(\varepsilon_{1}, \varepsilon_{2}, \varepsilon_{3}, \varepsilon_{6}, \varepsilon_{7}, \varepsilon_{8}, \varepsilon_{9}\right) \text {. }
\end{aligned}
$$

Then, the nonlinear system (15) with norm-bounded parametric uncertainties (17) and bounded disturbance (14) is robustly stabilizable by means of the saturated affine statefeedback control law (19) subject to constraint (18). Moreover, the matrix gain $\mathbf{K}$ of the control law (19) is

$$
\mathbf{K}=\mathbf{R S}^{-1} \text {. }
$$

Proof. The proof of this theorem was already achieved in the previous sections.

As noted in Theorem 2, the parameters $\gamma, \mu, \eta, \varepsilon_{m}, \rho, \bar{\delta}_{a}$, $\bar{\delta}_{b}, \bar{\delta}_{c}, \bar{\delta}_{d}$, and $\bar{\delta}_{e}$ should be fixed a priori to obtain a solution of LMIs (57)-(59). Thus, the computation complexity for solving these three LMIs is reduced. However, in this way, the design conservatism will increase and obtaining a solution becomes more difficult. Indeed, we should test several times the feasibility of these LMIs (57)-(59) with different values of all these fixed parameters. Therefore, in order to reduce the conservatism of the designed LMIs, we should consider these parameters as decision variables, which should be optimized numerically. To this end, we next present improved LMI conditions. We note that the parameter $\varepsilon_{m}$ can be selected as a decision variable to be minimized. Nevertheless, we will consider it as a predefined constant scalar, which will be fixed to be very small.

Remark 4. As noted in Remark 3, for a general case where $n_{u}>1$, we obtain the BMI (37) instead of the BMI (26). The linearization of the BMI stability condition (37) leads to the following LMI:

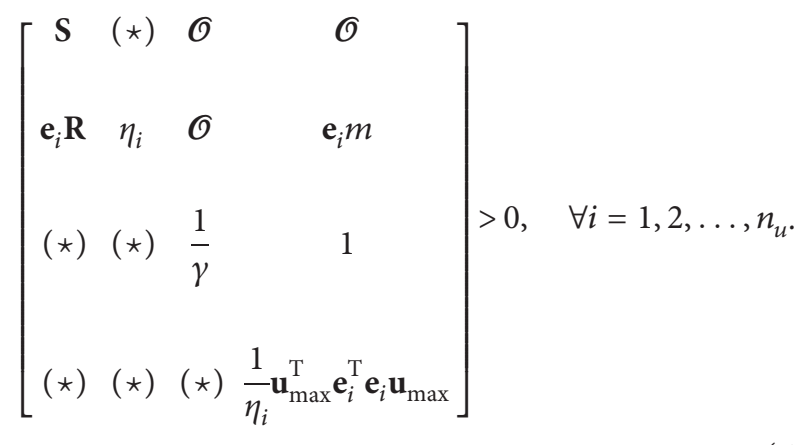

3.3. Enhanced LMI Stability Conditions. Our immediate concern is to convert the constant parameters $\gamma, \mu, \eta, \rho, \bar{\delta}_{a}$, $\bar{\delta}_{b}, \bar{\delta}_{c}, \bar{\delta}_{d}$, and $\bar{\delta}_{e}$ into decision variables. First of all, it is evident that the two parameters $\bar{\delta}_{c}$ and $\bar{\delta}_{d}$ presented in the LMI (57) are independent of other unknown variables. Then, they can be considered as decision variables. However, the two main difficulties lie on the one hand in the parameters $\gamma$, $\mu$, and $\eta$ and on the other hand in the three parameters $\bar{\delta}_{a}, \bar{\delta}_{b}$, $\bar{\delta}_{e}$, and $\rho$. Transformation of $\bar{\delta}_{a}, \bar{\delta}_{e}$, and $\rho$ is simple [12]. Furthermore, transformation of the parameters $\gamma$ and $\bar{\delta}_{b}$ requires a certain judicious mathematical manipulation. Note that the parameter $\gamma$ appears in both LMI (57) and LMI (58). However, transformation of the two remaining parameters $\mu$ and $\eta$ is quite difficult and perhaps impossible. To simplify computation, we can pose $\mu=\eta$. Hence, we state the following enhanced version of Theorem 2 .

Theorem 3. Assume that, for some positive parameters $\lambda$ and $\epsilon_{m}$ fixed a priori such that $\epsilon_{m} \ll 1$, there exist a symmetric matrix $\mathbf{S}$, a matrix $\mathbf{R}$, a gain $m$, and some scalars $\gamma, \alpha>0$, $\beta>0, \varphi>0, \epsilon_{1}, \epsilon_{2}, \epsilon_{3}, \epsilon_{4}, \epsilon_{5}, \epsilon_{6}, \epsilon_{7}, \epsilon_{8}, \epsilon_{9}, \bar{\delta}_{c}$, and $\bar{\delta}_{d}$ so that the 
following optimization problem with LMI constraints is feasible:

$$
\begin{aligned}
\operatorname{minimize} & \left(\varepsilon_{4}+\varepsilon_{5}+\varepsilon_{6}+\varepsilon_{9}\right)-\left(\alpha+\beta+\varphi+\bar{\delta}_{c}+\bar{\delta}_{d}+\rho\right) \\
\text { subject to } & {\left[\begin{array}{cccc}
\widehat{\mathbb{A}}_{4}+\lambda \mathbf{S} & \mathbf{B} m & \widehat{\mathbb{A}}_{5} & \mathcal{O} \\
(\star) & -\lambda \gamma & \mathcal{O} & \widehat{\mathbb{A}}_{7} \\
(\star) & (\star) & -\mathbb{A}_{6} & \mathcal{O} \\
(\star) & (\star) & (\star) & -\mathbb{A}_{8}
\end{array}\right]<0, }
\end{aligned}
$$

$$
\begin{gathered}
{\left[\begin{array}{ccc}
\mathbf{S} & \mathbf{R}^{\mathrm{T}} & \mathcal{O} \\
(\star) & \lambda & m \\
(\star) & (\star) & \frac{u_{\max }^{2}}{\lambda}-\gamma
\end{array}\right]>0,} \\
{\left[\begin{array}{cc}
\widehat{\mathbf{C}}^{\mathrm{T}} \widehat{\mathbf{C}}+2 \widehat{\mathbf{C}}^{\mathrm{T}} \mathbf{B} m-\varepsilon_{m} & m^{\mathrm{T}} \mathbf{B}^{\mathrm{T}} \\
(\star) & -\mathscr{I}
\end{array}\right]<0,}
\end{gathered}
$$

where

$$
\begin{aligned}
& \widehat{\mathbb{A}}_{4}=(\mathbf{A S}+\mathbf{B R})+(*)+\varepsilon_{1} \mathbf{C C}^{\mathrm{T}}+\varepsilon_{2} \mathbf{D D}^{\mathrm{T}}+\varepsilon_{3} \mathbf{E} \mathbf{E}^{\mathrm{T}} \\
& +\left(\alpha+\beta+\varphi+\varepsilon_{7}+\varepsilon_{8}\right) \mathbf{F F}^{\mathrm{T}} \text {, } \\
& \widehat{\mathbb{A}}_{5}=\left[\begin{array}{ll}
\mathbf{S F} & \mathbf{R}^{\mathrm{T}}
\end{array}\right] \text {, } \\
& \mathbb{A}_{6}=\operatorname{diag}\left(\varepsilon_{4}, \varepsilon_{5}\right) \text {, } \\
& \widehat{\mathbb{A}}_{7}=\left[\begin{array}{lllllll}
1 & 1 & 1 & m^{T} & \bar{\delta}_{c} & \bar{\delta}_{d} & \rho
\end{array}\right], \\
& \mathbb{A}_{8}=\operatorname{diag}\left(\varepsilon_{1}, \varepsilon_{2}, \varepsilon_{3}, \varepsilon_{6}, \varepsilon_{7}, \varepsilon_{8}, \varepsilon_{9}\right) \text {. }
\end{aligned}
$$

Then, the uncertain disturbed nonlinear system (14) is robustly stabilizable via the saturated affine state-feedback control law (18), with $\mathbf{K}=\mathbf{R S}^{-1}$, and

$$
\begin{aligned}
& \bar{\delta}_{a}=\sqrt{\frac{\alpha}{\varepsilon_{4}}}, \\
& \bar{\delta}_{b}=\sqrt{\frac{\beta}{\varepsilon_{5}+\varepsilon_{6}}}, \\
& \bar{\delta}_{e}=\sqrt{\frac{\varphi}{\varepsilon_{9}}}
\end{aligned}
$$

Proof. We first consider expression (29). Thus, by applying the Young relation, we can obtain another related condition instead of inequality (30):

$$
\begin{aligned}
\dot{V}(\mathbf{x}) & +\mu(V(\mathbf{x})-\gamma) \leq \mu \mathbf{x}^{\mathrm{T}} \mathbf{P} \mathbf{x}-\mu \gamma+2 \mathbf{x}^{\mathrm{T}} \mathbf{P} \overline{\mathbf{A}} \mathbf{x}+2 \mathbf{x}^{\mathrm{T}} \mathbf{P B} m \\
& +\varepsilon_{1} \mathbf{x}^{\mathrm{T}} \mathbf{P} \mathbf{C C}^{\mathrm{T}} \mathbf{P} \mathbf{x}+\varepsilon_{1}^{-1}+\varepsilon_{2}^{-1}+\varepsilon_{3}^{-1}+\varepsilon_{2} \mathbf{x}^{\mathrm{T}} \mathbf{P} \mathbf{D D}^{\mathrm{T}} \mathbf{P} \mathbf{x} \\
& +\varepsilon_{3} \mathbf{x}^{\mathrm{T}} \mathbf{P} \mathbf{E E}^{\mathrm{T}} \mathbf{P} \mathbf{x}+\left(\varepsilon_{4} \bar{\delta}_{a}^{2}+\left(\varepsilon_{5}+\varepsilon_{6}\right) \bar{\delta}_{b}^{2}+\varepsilon_{7}+\varepsilon_{8}+\varepsilon_{9} \bar{\delta}_{e}^{2}\right) \\
& +\mathbf{x}^{\mathrm{T}} \mathbf{P} \mathbf{F} \mathbf{F}^{\mathrm{T}} \mathbf{P} \mathbf{x}+\varepsilon_{4}^{-1} \mathbf{x}^{\mathrm{T}} \mathbf{F} \mathbf{F}^{\mathrm{T}} \mathbf{x}+\varepsilon_{5}^{-1} \mathbf{x}^{\mathrm{T}} \mathbf{K}^{\mathrm{T}} \mathbf{K} \mathbf{x}+\varepsilon_{6}^{-1} m^{\mathrm{T}} m \\
& +\varepsilon_{7}^{-1} \bar{\delta}_{c}^{2}+\varepsilon_{8}^{-1} \bar{\delta}_{d}^{2}+\varepsilon_{9}^{-1} \rho^{2}<0 .
\end{aligned}
$$

Let us introduce a new change of variables by posing $\alpha=\varepsilon_{4} \bar{\delta}_{a}^{2}, \beta=\left(\varepsilon_{5}+\varepsilon_{6}\right) \bar{\delta}_{b}^{2}$, and $\varphi=\varepsilon_{9} \bar{\delta}_{e}^{2}$. Then, according to the previous section, we obtain the LMI (64), in which $\alpha, \beta$, and $\varphi$ are three decision variables. Hence, once the values of $\alpha, \beta, \varphi, \varepsilon_{4}, \varepsilon_{5}, \varepsilon_{6}$, and $\varepsilon_{9}$ are obtained, we calculate $\bar{\delta}_{a}, \bar{\delta}_{b}$, and $\bar{\delta}_{e}$ according to the following relations: $\bar{\delta}_{a}=\sqrt{\alpha / \varepsilon_{4}}$, $\bar{\delta}_{b}=\sqrt{\beta /\left(\varepsilon_{5}+\varepsilon_{6}\right)}$, and $\bar{\delta}_{e}=\sqrt{\varphi / \varepsilon_{9}}$.

The remaining problem is the parameter $\gamma$, which appears in both LMI (57) and LMI (58). We premultiply and postmultiply LMI (58) by the matrix $\left[\begin{array}{cccc}\mathscr{I} & \mathcal{O} & \mathcal{O} & \mathscr{O} \\ (\star) & \mathscr{I} & \mathcal{O} & \mathcal{O} \\ (\star) & (\star) & \mathcal{O} & \mathscr{I} \\ (\star) & (\star) & (\star) & \mathcal{O}\end{array}\right]$ and then we obtain

$$
\left[\begin{array}{cccc}
\mathbf{S} & \mathbf{R}^{\mathrm{T}} & \mathcal{O} & \mathcal{O} \\
(\star) & \eta & m & \mathcal{O} \\
(\star) & (\star) & \frac{u_{\max }^{2}}{\eta} & 1 \\
(\star) & (\star) & (\star) & \frac{1}{\gamma}
\end{array}\right]>0 .
$$

By applying the Schur lemma, inequality (69) is equivalent to the LMI (64). Now, the parameter $\gamma$ is linear in the two LMIs (63) and (64).

In addition, the parameter $\mu$ appears in the LMI (57), whereas the parameter $\eta$ appears in the LMI (58). As noted before, it is difficult to transform $\mu$ and $\eta$ into two decision variables. Then, to simplify the computation procedure, we can pose $\mu=\eta=\lambda$.

The objective is to look for maximum values of the uncertainty bounds $\bar{\delta}_{a}, \bar{\delta}_{b}, \bar{\delta}_{c}, \bar{\delta}_{d}$, and $\bar{\delta}_{e}$ and the disturbance bound $\rho$. The three parameters $\bar{\delta}_{c}, \bar{\delta}_{d}$, and $\rho$ appear directly in the LMI (63) (matrix $\widehat{\mathbb{A}}_{7}$ ). Then, these parameters should be maximized. However, the variables $\bar{\delta}_{a}, \bar{\delta}_{b}$, and $\bar{\delta}_{e}$ are incorporated, respectively, in the parameters $\alpha, \beta$, and $\varphi: \alpha=\varepsilon_{4} \bar{\delta}_{a}^{2}$, $\beta=\left(\varepsilon_{5}+\varepsilon_{6}\right) \bar{\delta}_{b}^{2}$, and $\varphi=\varepsilon_{9} \bar{\delta}_{e}^{2}$. Then, to maximize $\bar{\delta}_{a}, \bar{\delta}_{b}$, and $\bar{\delta}_{e}$, we should maximize $\alpha, \beta$, and $\varphi$. Moreover, as $\alpha(\operatorname{resp} . \beta, \varphi)$ depends on $\varepsilon_{4}$ (resp. $\varepsilon_{5}$ and $\varepsilon_{6}, \varepsilon_{9}$ ), we should minimize $\varepsilon_{4}, \varepsilon_{5}, \varepsilon_{6}$, and $\varepsilon_{9}$ in order to ensure maximized values of $\bar{\delta}_{a}, \bar{\delta}_{b}$, and $\bar{\delta}_{e}$. We emphasize that, in order to obtain a minimization objective function that can be handled with the LMI toolbox, we should transform the maximization problem of the variables $\alpha, \beta, \varphi, \bar{\delta}_{c}$, $\bar{\delta}_{d}$, and $\rho$ into a minimization problem of the quantity $-(\alpha+$ $\left.\beta+\varphi+\bar{\delta}_{c}+\bar{\delta}_{d}+\rho\right)$. This completes the proof of Theorem 3 .

It is worth to mention that Theorem 3 offers a wider choice of invariant ellipsoids $\varepsilon(\mathbf{P}, \gamma)$ (as $\gamma$ varies) for optimization and will lead to less conservative estimation of the domain of attraction.

In addition, we stress that, in Theorem 3, we imposed the two free parameters $\mu$ and $\eta$ in Theorem 2 to be equal, i.e., $\mu=\eta=\lambda$, in order to obtain an LMI with a single parameter $\lambda$. Nevertheless, this choice, which is the only solution to simplify computation, injects some degree of conservatism in the LMI conditions (63)-(65). Furthermore, the parameter $\lambda$ is the only one that should be fixed a priori to obtain a solution of the optimization problem in Theorem 3. 


\section{Estimation of the Domain of Attraction}

With all the ellipsoids satisfying the set invariance condition (21), we would like to choose from among them the largest one to get at least an estimation of the domain of attraction. Certain criteria are used in the literature to maximize the size of the attractive invariant ellipsoid $\varepsilon(\mathbf{P}, \gamma)$, such as maximization of its volume and maximization of the sum of its semiaxis $[5,9,18,21,27,47,48,52]$. Actually, maximization of the volume of an ellipsoid is equivalent to minimization of the determinant of the Lyapunov matrix $\mathbf{P}$, and maximization of the sum of its semiaxis is equivalent to minimization of the trace of the matrix $\mathbf{P}$ [9].

In this part, our main goal is to find a robust saturated affine state-feedback control law (18) associated to the largest invariant ellipsoid $\varepsilon_{\star}\left(\mathbf{P}_{\star}, \gamma_{\star}\right)$. Such largest invariant ellipsoid will be found for desired values of the maximum bounds of the parametric uncertainties $\bar{\delta}_{a}, \bar{\delta}_{b}, \bar{\delta}_{c}, \bar{\delta}_{d}$, and $\bar{\delta}_{e}$ and also the maximum bound of the disturbance $\rho$. Thus, we will use LMI (63) (or systematically LMI (57)) for predefined values of these maximum bounds. However, we should also use the two LMIs (64) and (65) to look for a maximum value of the parameter $\gamma$ and hence the associated gains $\mathbf{K}$ and $m$.

Next, the size of the attractive invariant ellipsoid $\varepsilon(\mathbf{P}, \gamma)$ is measured through the sum of its semiaxis. For this subject, we introduce the following theorem.

Theorem 4. The largest attractive invariant ellipsoid of the nonlinear system (15) with bounded disturbance (14) and norm-bounded parametric uncertainties (17), subject to the saturated affine state-feedback control law defined by expression (19) and constraint (18), is the ellipsoid $\varepsilon(\mathbf{P}, \gamma)$, for a fixed positive scalar $\varepsilon_{m} \ll 1$ and some positive parameter $\lambda$ known a priori, where $\mathbf{P}=\mathbf{S}^{-1}$ and $\gamma$, together with $\varepsilon_{1}, \varepsilon_{2}, \varepsilon_{3}$, $\varepsilon_{4}, \varepsilon_{5}, \varepsilon_{6}, \varepsilon_{7}, \varepsilon_{8}$, and $\varepsilon_{9}$, are a solution of the following LMIbased optimization problem:

$$
\begin{aligned}
& \operatorname{minimize}(-\operatorname{trace}(\mathbf{S})-\gamma) \text {, } \\
& {\left[\begin{array}{cccc}
\mathbb{A}_{4}+\lambda \mathbf{S} & \mathbf{B} m & \mathbb{A}_{5} & \mathcal{O} \\
(\star) & -\lambda \gamma & \mathcal{O} & \mathbb{A}_{7} \\
(\star) & (\star) & -\mathbb{A}_{6} & \mathcal{O} \\
(\star) & (\star) & (\star) & -\mathbb{A}_{8}
\end{array}\right]<0,} \\
& {\left[\begin{array}{ccc}
\mathbf{S} & \mathbf{R}^{\mathrm{T}} & \mathcal{O} \\
(\star) & \lambda & m \\
(\star) & (\star) & \frac{u_{\max }^{2}}{\lambda}-\gamma
\end{array}\right]>0} \\
& {\left[\begin{array}{cc}
\widehat{\mathbf{C}}^{\mathrm{T}} \widehat{\mathbf{C}}+2 \widehat{\mathbf{C}}^{\mathrm{T}} \mathbf{B} m-\varepsilon_{m} & m^{\mathrm{T}} \mathbf{B}^{\mathrm{T}} \\
(\star) & -\mathscr{I}
\end{array}\right]<0 .}
\end{aligned}
$$

Proof. In expression (21), the attractive invariant ellipsoid $\mathscr{E}(\mathbf{P}, \gamma)$ can be recast as

$$
\varepsilon\left(\frac{\mathbf{P}}{\gamma}, 1\right)=\left\{\mathbf{x} \in \mathbb{R}^{2}: \mathbf{x}^{\mathrm{T}} \frac{\mathbf{P}}{\gamma} \mathbf{x} \leq 1\right\} .
$$

We emphasize that the trace of the matrix $\mathbf{P} / \gamma$ is equal to the sum of the semiaxis of the invariant ellipsoid $\varepsilon(\mathbf{P} / \gamma, 1)$ for some fixed $\gamma$. Hence, the size (the length of the semiaxis) of $\varepsilon(\mathbf{P} / \gamma, 1)$ increases as the trace of the matrix $\mathbf{P} / \gamma$ decreases. As $\mathbf{P}=\mathbf{S}^{-1}, \mathbf{P} / \gamma=(\gamma \mathbf{S})^{-1}$. Therefore, maximization of the size of $\varepsilon\left((\gamma \mathbf{S})^{-1}, 1\right)$ is equivalent to the maximization of the trace of the matrix $\gamma \mathbf{S}$. As a result, to guarantee the largest attractive invariant ellipsoid $\varepsilon\left((\gamma \mathbf{S})^{-1}, 1\right)$, we should maximize the parameter $\gamma$ and also the trace of the matrix $\mathbf{S}$. Notice that maximizing $\gamma$ and $\operatorname{trace}(\mathbf{S})$ is equivalent to minimizing $(-\operatorname{trace}(\mathbf{S})-\gamma)$. This completes the proof of Theorem 4.

It is worth to note that, for each fixed value of the parameter $\lambda$, the optimization problem in Theorem 4 provides a solution associated to an attractive invariant ellipsoid $\varepsilon(\mathbf{P}, \gamma)$ with a (local) largest size, which is obtained for such $\lambda$. Therefore, in order to obtain the global maximum, we should vary the parameter $\lambda, 0<\lambda<\infty$. To this end, the best manner is the use of the gridding method [68]. This method consists in making a new change of variable by defining $\kappa=$ $\lambda /(1+\lambda)$ and then $\lambda=\kappa /(1-\kappa)$. We know that $\lambda>0$ if and only if $\kappa \in] 0,1[$. Then, we assign a uniform subdivision of the interval ] 0,1 [ and we solve the optimization problem in Theorem 4 for each value of this subdivision. Once this interval is covered, we look for the maximum value between all the obtained results. This maximum value corresponds to the largest invariant ellipsoid, which was obtained for some $\kappa_{\star}$ and then for some optimal values of the parameter $\lambda$, saying $\lambda_{\star}$.

In fact, finding the optimal value $\lambda_{\star}$ and then the largest invariant attractive ellipsoid $\varepsilon_{\star}$ takes a lot of time. Indeed, suppose that we uniformly subdivide the interval ] $0,1[$, in which the parameter $\kappa$ varies, and then we take $N_{\kappa}$ values. Thus, for each value of $\kappa$, we run the optimization algorithm in Theorem 4. Let us assume that a single run takes $t_{\kappa}$. Hence, the total simulation time in order to obtain the largest ellipsoid $\varepsilon_{\star}$ is about $t_{c} \approx N_{\kappa} \times t_{\kappa}$.

Remark 5. In several research works presented in the literature, the computation of the largest attractive invariant ellipsoid $\varepsilon_{\star}\left(\mathbf{P}_{\star}, \gamma_{\star}\right)$ is achieved mainly in two steps:

(1) Hunt for an invariant ellipsoid $\varepsilon(\mathbf{P}, 1)$ by obtaining the matrix $\mathbf{P}$

(2) With such $\mathbf{P}$, maximize the value of the parameter $\gamma$

Actually, these two steps are realized via two optimization algorithms. Compared with this hunting procedure, our proposed design of the saturated feedback control law guarantees the determination of the largest invariant ellipsoid $\varepsilon_{\star}\left(\mathbf{P}_{\star}, \gamma_{\star}\right)$ in only one step and then with only one optimization algorithm. 


\section{Simulation Results}

In this section, we provide some simulation results to demonstrate the effectiveness of our developed method for the synthesis of the saturated affine state-feedback control law (19) for the robust stabilization of the disturbed uncertain nonlinear dynamics (15) of the pitch model of the helicopter. Moreover, we aim at identifying and analyzing the largest attractive invariant ellipsoid $\varepsilon_{\star}\left(\mathbf{P}_{\star}, \gamma_{\star}\right)$ in order to obtain an estimation of the domain of attraction. Thus, in the sequel, we take two values of the saturation level in (18) of the control law $u$ : $u_{\max }=1$ and $u_{\max }=10$. Moreover, we fix the parameter $\varepsilon_{m}=10^{-5}$.

The values of the parameters figured in the nonlinear dynamics (12) or of the pitch model of the helicopter are as follows [55-57]: $I_{y y}=0.0283\left(\mathrm{~kg} / \mathrm{m}^{2}\right), m_{\text {heli }}=0.9941(\mathrm{Kg})$, $l_{c g x}=0.0134(\mathrm{~m}), \quad l_{c g z}=0.0289(\mathrm{~m}), \quad F_{v M}=0.0041$ $(\mathrm{Nm} / \mathrm{rad} / \mathrm{s}), g=9.81\left(\mathrm{~m} / \mathrm{s}^{2}\right)$, and $s=0.0057\left(\mathrm{~m} / \mathrm{s}^{2}\right)$.

Remark 6. The choice of the parameters of the maximum value of the control input $u, u_{\max }$, is based on two facts: (1) in the literature, the common choice of the saturation limit is $u_{\max }=1$ according to the invariance sets' concept and (2) in the present work, we have considered a general case of the actuator saturation limit in the development of LMI constraints and hence in the design of the state-feedback controller in order to show the effect of the saturation limit $u_{\max }$ on the largeness of the invariant attractive ellipsoid.

Thus, in our investigation, we selected two values of the saturation limit: $u_{\max }=1$ (which is the classical choice) and $u_{\max }=10$. We can also choose another value of such saturation limit such as $u_{\max }=24$, and we will obtain similar results.

Actually, the saturation limits are the intrinsic requirement of the physical actuators. Thus, the adopted different values of the $u_{\max }$ will be used in the sequel just to show the feasibility and the conservativeness of the proposed design method of the two gains of the affine state-feedback controller and also for the computation of the largest domain of attraction in different situations.

Remark 7. It is worth to mention that for the computation of the solutions of the LMIs in Theorem 2 and the LMI-based optimization problem in Theorems 3 and 4, we use the LMI toolbox of MATLAB. We can also use the toolbox YALMIP [10] in order to solve these LMI problems. Moreover, we can use it to (locally) solve some BMI problems using the bisection method.

5.1. Numerical Results. The optimization problem subject to LMI constraints in Theorem 3 provides numerical results illustrated in Table 1 for the saturation level $u_{\max }=1$ and for different values of the fixed parameter $\lambda$. In this table, we provide the two gains, $\mathbf{K}$ and $m$, of the saturated affine statefeedback control law $u$, the maximum bounds of the parametric uncertainties, i.e., $\bar{\delta}_{a}, \bar{\delta}_{b}, \bar{\delta}_{c}, \bar{\delta}_{d}$, and $\bar{\delta}_{e}$, the maximum bound of the disturbance $w$, i.e., $\rho$, and the parameter $\gamma$. It is obvious that the three uncertainty bounds, $\bar{\delta}_{a}, \bar{\delta}_{b}$, and $\bar{\delta}_{e}$, are very small, around $10^{-4}$. The two parameters, $\bar{\delta}_{c}$ and $\bar{\delta}_{d}$, are found to be equal and decrease slightly as $\lambda$ increases. However, the value of the allowable disturbance bound $\rho$ is found to be very high, about $6 \times 10^{3}$.

We emphasize that the obtained results for the allowable maximum bounds of the parametric uncertainties, $\bar{\delta}_{a}, \bar{\delta}_{b}$, and $\bar{\delta}_{e}$, are dissatisfying (they are found to be very small). Moreover, the maximum disturbance bound is found to be very high. It is worth noting that the optimization algorithm in Theorem 3 is characterized by an objective function that depends on 10 parameters $\varepsilon_{4}, \varepsilon_{5}, \varepsilon_{6}, \varepsilon_{9}, \alpha, \beta, \varphi, \bar{\delta}_{c}, \bar{\delta}_{d}$, and $\rho$, which are optimized together. Then, in order to improve the obtained results, we will fix in the next the parameter $\rho$ and the remaining ones will be optimized. As discussed in the beginning of this paper, in the second section, the external disturbance $w$ can represent the turbulent moments on the helicopter. Without loss of generality, we will fix $\rho=10$.

Thus, for $\rho=10$, the optimization algorithm subject to LMI constraints in Theorem 3 gives numerical results illustrated in Table 2 for the case $u_{\max }=1$ and in Table 3 for $u_{\max }=10$. As in Table 1, we provide, for different values of the parameter $\lambda$, the gain matrix $\mathbf{K}$, the gain constant $m$, the maximum bounds of the parametric uncertainties $\bar{\delta}_{a}, \bar{\delta}_{b}, \bar{\delta}_{c}$, $\bar{\delta}_{d}$, and $\bar{\delta}_{e}$, and the parameter $\gamma$.

We note from Tables 2 and 3 that the optimization problem provides identical values for the two uncertainty bounds $\bar{\delta}_{c}$ and $\bar{\delta}_{d}$. Moreover, for all values of $\lambda$, the value of the gain $m$ is about 0.0133 as in Table 1 . In addition, it is evident that, for the same value of parameter $\lambda$, we have almost the same gain matrix K. Furthermore, as $\lambda$ increases, the size of the gain matrix $\mathbf{K}$ and the uncertainty bounds, $\bar{\delta}_{a}, \bar{\delta}_{b}$, and $\bar{\delta}_{e}$, increase. For $u_{\max }=1, \bar{\delta}_{c}$ is found to be around $10^{-3}$, whereas for $u_{\max }=10, \bar{\delta}_{c}$ is about 14 . In addition, it is evident that the values of the maximum bounds of the parametric uncertainties $\bar{\delta}_{a}, \bar{\delta}_{b}$, and $\bar{\delta}_{e}$ are bigger than those obtained in Table 1 since the parameter $\rho$ is fixed here.

We can also observe from Tables 2 and 3 that, as the parameter $\lambda$ increases, the value of the parameter $\gamma$ decreases. Actually, we can note that $\gamma \approx u_{\max }^{2} / \lambda$. This shows that the size of the attractive invariant ellipsoid, which depends on $\gamma$, varies with respect to the value of the saturation level $u_{\max }$.

\subsection{Computation of the Largest Attractive Invariant Ellipsoid.}

To obtain the largest attractive invariant ellipsoid $\varepsilon_{\star}\left(\mathbf{P}_{\star}, \gamma_{\star}\right)$, we solve the optimization problem in Theorem 4 . Thus, in order to obtain the global largest ellipsoid $\varepsilon_{\star}\left(\mathbf{P}_{\star}, \gamma_{\star}\right)$, we should vary the free parameter $\lambda$ according to the gridding method as noted before. Moreover, in this optimization problem, we fix the bounds of the parametric uncertainties and we take a common value, $\bar{\delta}$, for all these bounds like so: for $u_{\max }=1$, we take $\bar{\delta}_{a}=\cdots=\bar{\delta}_{e}=\bar{\delta}=1$, whereas for $u_{\max }=10$, we fix $\bar{\delta}_{a}=\cdots=\bar{\delta}_{e}=\bar{\delta}=10$. Actually, with $\bar{\delta}=$ 10 and $u_{\max }=1$, the optimization problem in Theorem 4 is unfeasible. It was found unfeasible also for all $\bar{\delta} \geq 2$ and $u_{\max }=1$. Then, we minimized the uncertainty bound to 
TABLE 1: Numerical simulation results obtained using the optimization algorithm in Theorem 3 for different values of the parameter $\lambda$ and for $u_{\max }=1$.

\begin{tabular}{|c|c|c|c|c|c|c|c|}
\hline & \multicolumn{2}{|c|}{$\lambda=0.01$} & \multicolumn{2}{|c|}{$\lambda=0.1$} & \multirow{2}{*}{$\begin{array}{c}\lambda=1 \\
{\left[\begin{array}{l}-0.0204 \\
-0.0437\end{array}\right]}\end{array}$} & \multirow{2}{*}{$\left.\begin{array}{c}\lambda=10 \\
-2.3360 \\
-0.5053\end{array}\right]$} & \multirow{2}{*}{$\begin{array}{c}\lambda=100 \\
{\left[\begin{array}{c}-236.7854 \\
-5.1249\end{array}\right]}\end{array}$} \\
\hline $\mathbf{K}^{\mathrm{T}}$ & $10^{-3} \times$ & $\begin{array}{l}-0.0372 \\
-0.2566\end{array}$ & $10^{-3} \times$ & {$\left[\begin{array}{l}-0.3786 \\
-2.6152\end{array}\right.$} & & & \\
\hline$m$ & \multicolumn{2}{|c|}{0.013321} & \multicolumn{2}{|c|}{0.013321} & 0.013321 & 0.013321 & 0.013321 \\
\hline $\bar{\delta}_{a}$ & \multicolumn{2}{|c|}{$10^{-4} \times 4.5975$} & \multicolumn{2}{|c|}{$10^{-4} \times 4.6197$} & $10^{-4} \times 3.0634$ & $10^{-4} \times 2.7950$ & $10^{-4} \times 2.7613$ \\
\hline $\bar{\delta}_{b}^{u}$ & \multicolumn{2}{|c|}{$10^{-4} \times 3.2525$} & \multicolumn{2}{|c|}{$10^{-4} \times 3.2666$} & $10^{-4} \times 2.1641$ & $10^{-4} \times 1.9764$ & $10^{-4} \times 1.9526$ \\
\hline $\bar{\delta}_{c}=\bar{\delta}_{d}$ & \multirow{2}{*}{\multicolumn{2}{|c|}{$\begin{array}{c}3.6314 \\
10^{-4} \times 4.5944\end{array}$}} & \multirow{2}{*}{\multicolumn{2}{|c|}{$\begin{array}{c}3.6515 \\
10^{-4} \times 4.6215\end{array}$}} & 2.2531 & 1.9868 & 1.9407 \\
\hline $\bar{\delta}_{e}$ & & & & & $10^{-4} \times 3.0796$ & $10^{-4} \times 2.7990$ & $10^{-4} \times 2.7613$ \\
\hline$\rho$ & \multicolumn{2}{|c|}{$10^{3} \times 6.4069$} & \multicolumn{2}{|c|}{$10^{3} \times 6.4673$} & $10^{3} \times 6.0719$ & $10^{3} \times 5.4419$ & $10^{3} \times 5.7484$ \\
\hline$\gamma$ & \multicolumn{2}{|c|}{81.4039} & \multicolumn{2}{|c|}{8.1419} & 0.8527 & 0.0863 & 0.0087 \\
\hline
\end{tabular}

TABle 2: Numerical simulation results using Theorem 3 for different values of the parameter $\lambda$ and for $\rho=10$ and $u_{\max }=1$.

\begin{tabular}{lcccccc}
\hline & $\lambda=0.01$ & $\lambda=0.1$ & $\lambda=1$ & $\lambda=10$ & $\lambda=100$ & $\lambda=200$ \\
\hline $\mathbf{K}^{\mathrm{T}}$ & {$\left[\begin{array}{l}-0.00089 \\
-0.00503\end{array}\right]$} & {$\left[\begin{array}{l}-0.00298 \\
-0.02858\end{array}\right]$} & {$\left[\begin{array}{l}-0.09235 \\
-0.11705\end{array}\right]$} & {$\left[\begin{array}{c}-3.6488 \\
-0.6523\end{array}\right]$} & {$\left[\begin{array}{c}-298.7673 \\
-5.8965\end{array}\right]$} & 0.013251 \\
$m$ & 0.013275 & 0.013283 & 0.013268 & 0.013243 & 5.4867 & 0.013262 \\
$\bar{\delta}_{a}$ & 0.0067 & 0.0181 & 0.7729 & 1.9138 & 2.6496 & 7.7133 \\
$\bar{\delta}_{b}$ & 0.0800 & 0.1020 & 2.2687 & 2.6586 & 0.0022 & $0.0117]$ \\
$\bar{\delta}_{c}=\bar{\delta}_{d}$ & 0.0048 & 0.0029 & 0.0043 & 0.0011 & 0.7459 & 0.7475 \\
$\bar{\delta}_{e}$ & 0.0112 & 0.0155 & 0.4577 & 0.7132 & 0.0099 & 0.0049 \\
$\gamma$ & 99.1571 & 9.9099 & 0.9893 & 0.0986 & 2.1796 \\
\hline
\end{tabular}

TABle 3: Numerical simulation results using Theorem 3 for different values of the parameter $\lambda$ and for $\rho=10$ and $u_{\max }=10$.

\begin{tabular}{lcccccc}
\hline & $\lambda=0.01$ & $\lambda=0.1$ & $\lambda=1$ & $\lambda=10$ & $\lambda=100$ & $\lambda=200$ \\
\hline $\mathbf{K}^{\mathrm{T}}$ & {$\left[\begin{array}{l}-0.00048 \\
-0.04438\end{array}\right]$} & {$\left[\begin{array}{l}-0.00581 \\
-0.05694\end{array}\right]$} & {$\left[\begin{array}{l}-0.0917 \\
-0.1166\end{array}\right]$} & {$\left[\begin{array}{c}-3.6420 \\
-0.6515\end{array}\right]$} & {$\left[\begin{array}{c}-297.9499 \\
-5.8866\end{array}\right]$} & 0.013321 \\
$m$ & 0.01327 & 0.013319 & 0.013316 & 0.013320 & 6.8305 & 0.013321 \\
$\bar{\delta}_{a}$ & 0.0321 & 0.8835 & 1.2411 & 2.4613 & 3.3309 & 9.3191 \\
$\bar{\delta}_{b}$ & 0.1500 & 3.6262 & 3.6585 & 3.4313 & 1.1761 \\
$\bar{\delta}_{c}=\bar{\delta}_{d}$ & 9.9995 & 14.5636 & 14.3332 & 14.3665 & 6.3217 & 14.7111 \\
$\bar{\delta}_{e}$ & 0.0360 & 2.1881 & 4.8950 & 6.0531 & 6.1668 \\
$\gamma$ & 9990.7 & 997.292 & 99.0173 & 9.8937 & 0.9851 & 0.4922 \\
\hline
\end{tabular}

obtain a feasible solution. This will be demonstrated and discussed next.

Figure 2 reveals evolution of the trace of the matrix $\gamma \mathbf{S}$, or systematically the sum of the semiaxis of the invariant ellipsoid $\varepsilon\left(\mathbf{S}^{-1}, \gamma\right)$, as the parameter $\lambda$ varies. It is obvious from Figure 2 that, by sweeping through $\lambda$, we obtain the maximum trace of the matrix $\gamma \mathbf{S}$ for the two cases $u_{\max }=1$ and $u_{\max }=10$. Such maximum trace corresponds to the largest invariant ellipsoid $\varepsilon_{\star}\left(\mathbf{P}_{\star}, \gamma_{\star}\right)$. Obtained results are summarized in Table 4 . In this table, we provide the parameters, $\mathbf{P}_{\star}$ and $\gamma_{\star}$, of the largest attractive invariant ellipsoid $\varepsilon_{\star}\left(\mathbf{P}_{\star}, \gamma_{\star}\right)$, the associated feedback gains, $\mathbf{K}_{\star}$ and $m_{\star}$, of the saturated affine state-feedback control law $u$, the nominal value, $\lambda_{\star}$, of the free parameter $\lambda$, and the size of the attractive invariant ellipsoid $\varepsilon_{\star}\left(\mathbf{P}_{\star}, \gamma_{\star}\right)$. According to Figure 2 and Table 4 , in the first case $u_{\max }=1$, the size of the largest invariant ellipsoid is about 1.7906 . However, in the second case $u_{\max }=10$, such ellipsoid has a size about 46.5870 . We recall that, for $u_{\max }=1$, we have chosen $\bar{\delta}=1$ and for $u_{\max }=10$, we have adopted $\bar{\delta}=10$.

Our objective now is to see the effect of the maximum bound of the different parametric uncertainties $\bar{\delta}_{a}, \bar{\delta}_{b}, \bar{\delta}_{c}, \bar{\delta}_{d}$, and $\bar{\delta}_{e}$ and also the saturation limit $u_{\max }$ on the size of the attractive invariant ellipsoid $\varepsilon(\mathbf{P}, \gamma)$. To do this, we used the optimization algorithm in Theorem 4 and we varied the parameter $\lambda$ as mentioned previously. This task was achieved for a single value of the maximum uncertainty bound, saying $\delta_{\max }=\bar{\delta}_{a}=\cdots=\bar{\delta}_{e}$. Thus, we obtained the largest ellipsoid $\varepsilon_{\star}\left(\mathbf{P}_{\star}, \gamma_{\star}\right)$. Now, we will repeat the same procedure by also varying the parameter $\delta_{\max }$ and fixing the saturation level $u_{\max }$. Figure 3 reveals obtained results for $u_{\max }=1$ and $u_{\max }=10$. We stress first that, for $u_{\max }=1$, the allowable maximum bound of the parametric uncertainty allowing to obtain an invariant ellipsoid $\varepsilon_{\star}\left(\mathbf{P}_{\star}, \gamma_{\star}\right)$ is $\delta_{\max }=1.06$. However, for $u_{\max }=10$, this value of $\delta_{\max }$ increases considerably and reaches the limit 12.05 . 


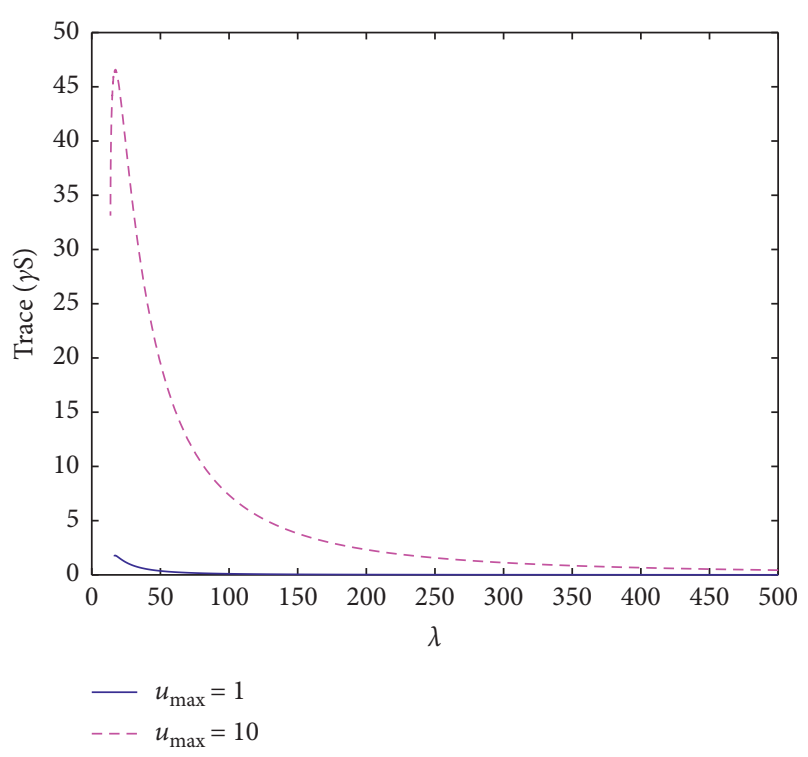

Figure 2: Variation of the trace of the matrix $\gamma \mathbf{S}$ with respect to the parameter $\lambda$ and for two different values of the saturation level: $u_{\max }=1$ and $u_{\max }=10$. Here, for $u_{\max }=1$ (resp. $u_{\max }=10$ ), we have adopted $\bar{\delta}=1$ (resp. $\bar{\delta}=10$ ). Moreover, in the two cases, we have fixed $\rho=10$.

Moreover, it is obvious that, for each value of $u_{\max }$, the size of the largest ellipsoid increases rapidly as $\delta_{\max }$ decreases. A tiny decrease of the uncertainty bound $\delta_{\max }$ leads to a very important increase in the size of the ellipsoid $\varepsilon_{\star}\left(\mathbf{P}_{\star}, \gamma_{\star}\right)$. For example, for $u_{\max }=1$ and for $\delta_{\max }=0.5$, the size is about $10^{3}$ and for $\delta_{\max }=0.1$, the size is around $10^{9}$. Similarly, for the saturation limit $u_{\max }=10$ and for $\delta_{\max }=0.5$, the size is around $10^{8}$ and for $\delta_{\max }=1.5$, the size is around $10^{5}$.

Furthermore, it is clear that the size of the largest invariant ellipsoid decreases as $\delta_{\max }$ increases. The ellipsoid becomes smaller as the uncertainty bound $\delta_{\max }$ approaches its feasible limit (i.e., the value 1.06 for $u_{\max }=1$ and 12.05 for $\left.u_{\max }=10\right)$.

In addition, we remark that, for the same value of uncertainty bound $\delta_{\max }$, the size of the largest ellipsoid $\varepsilon_{\star}\left(\mathbf{P}_{\star}, \gamma_{\star}\right)$ increases as the saturation limit $u_{\max }$ increases (as noted previously for $\delta_{\max }=0.5$ ).

Using numerical results illustrated in Table 4, we plotted in Figure 4 the corresponding largest attractive invariant ellipsoids $\varepsilon_{\star}\left(\mathbf{P}_{\star}, \gamma_{\star}\right)$ for the two cases $u_{\max }=1$ and $u_{\max }=10$. In Figure 4, the two inclined lines (colored in pink and green) correspond to the bounds of the actuator saturation (20), which are defined as $\mathbf{K x}+m= \pm u_{\max }$. The lower line (colored in pink) is depicted for $\mathbf{K x}+m=u_{\max }$.

It is obvious from Figure 4 that, in the two cases, the largest invariant ellipsoid $\varepsilon_{\star}\left(\mathbf{P}_{\star}, \gamma_{\star}\right)$ is contained in the region $\mathscr{L}\left(\mathbf{K}, \mathbf{m}, \mathbf{u}_{\max }\right)$, where $|\mathbf{K} \mathbf{x}+m| \leq u_{\max }$. Therefore, the constraints on the affine state-feedback control law given in (18) are well respected.

In the end of this part, we can note the two following statements:
TABle 4: Numerical results using Theorem 4 corresponding to the largest attractive invariant ellipsoid $\varepsilon_{\star}\left(\mathbf{P}_{\star}, \gamma_{\star}\right)$ for $u_{\max }=1$ and $u_{\max }=10$.

\begin{tabular}{lcc}
\hline & $u_{\max }=1$ and $\bar{\delta}=1$ & $u_{\max }=10$ and $\bar{\delta}=10$ \\
\hline $\mathbf{P}_{\star}$ & {$\left[\begin{array}{cc}10.1505 & 0.5523 \\
0.5523 & 0.0628\end{array}\right]$} & {$\left[\begin{array}{cc}64.4531 & 2.1626 \\
2.1626 & 0.1963\end{array}\right]$} \\
$\mathbf{K}_{\star}$ & {$\left[\begin{array}{c}-9.0604 \\
-1.0242 \\
0.013235\end{array}\right.$} & {$\left[\begin{array}{c}-20.3362 \\
-1.8458 \\
0.013303\end{array}\right]$} \\
$m_{\star}$ & 16.9211 & 17.3824 \\
$\lambda_{\star}$ & 0.0583 & 5.7446 \\
$\gamma_{\star}$ & 1.7906 & 46.5870 \\
$\operatorname{trace}\left(\gamma_{\star} \mathbf{S}_{\star}\right)$ &
\end{tabular}

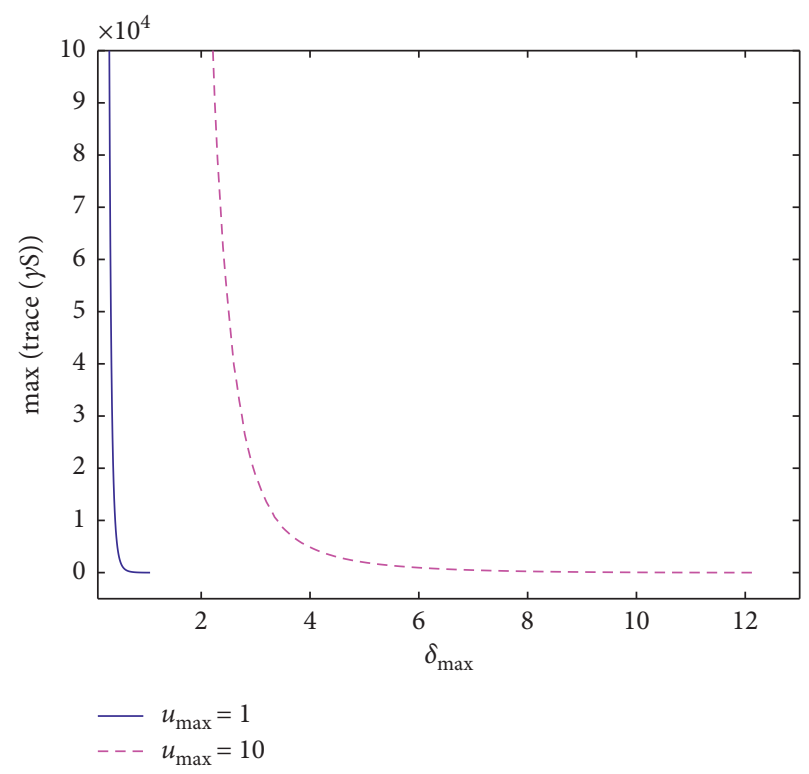

FIgURE 3: Variation of the size of the largest attractive invariant ellipsoid $\varepsilon_{\star}\left(\mathbf{P}_{\star}, \gamma_{\star}\right)$ with respect to the variation of the allowable maximum bound of the parametric uncertainties for the two cases: $u_{\max }=1$ and $u_{\max }=10$. Here, we have fixed $\bar{\delta}_{a}=\cdots=\bar{\delta}_{e}=\delta_{\max }$, with always $\rho=10$.

(1) For a constant saturation limit $u_{\max }$, an increase of the maximum bound of the parametric uncertainties leads to the decrease of the largeness of the attractive invariant ellipsoid.

(2) For a constant maximum bound of the uncertainty, an increase of the saturation limit $u_{\max }$ causes the attractive invariant ellipsoid to become larger.

Remark 8. In some research papers, the definition of the attractive invariant ellipsoid was chosen to be as follows:

$$
\dot{\varepsilon}(\mathbf{P})=\left\{\mathbf{x} \in \mathbb{R}^{n_{x}}: \mathbf{x}^{\mathrm{T}} \mathbf{P} \mathbf{x} \leq 1\right\} .
$$

This set $\dot{\varepsilon}(\mathbf{P})$ is equivalent to that adopted in the present work in (21), that is, $\varepsilon(\mathbf{P}, 1)$. Hence, we have $\gamma=1$. Our immediate concern is to show that the adopted ellipsoid (21) with a free parameter $\gamma$ gives less conservative results 


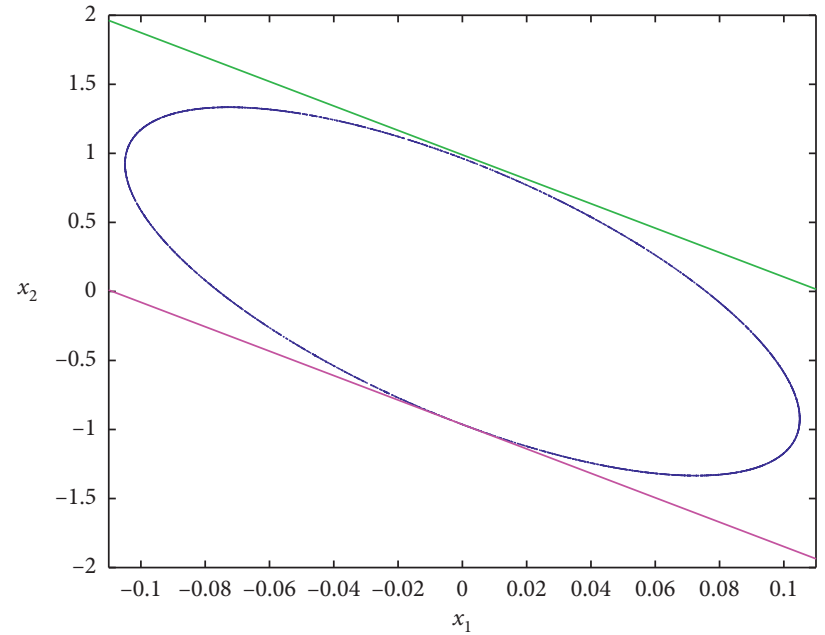

(a)

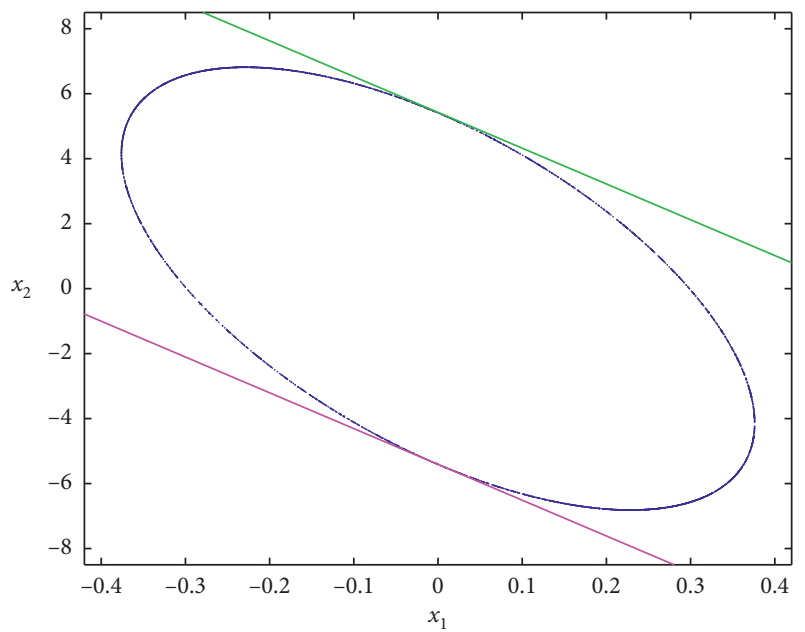

(b)

FIGURE 4: Largest attractive invariant ellipsoids $\varepsilon_{\star}\left(\mathbf{P}_{\star}, \gamma_{\star}\right)$ computed for two different values of $u_{\max }$.

compared with the ellipsoid (72). We use the same optimization algorithm in Theorem 4 where in the present case, we fix the parameter $\gamma$ as a constant one and then we take $\gamma=1$. Table 5 shows the numerical results obtained for a fixed parameter $\gamma=1$. Notice that the symbol (!) in Table 5 means that the LMI-based optimization problem in Theorem 4 is unfeasible. To realize a comparison from conservatism point of view, we adopt the same parameters in Table 4. As noted previously, the maximum bound of the disturbance is fixed to $\rho=10$. It is clear from Table 5 that, for the first case $u_{\max }=1$ and $\bar{\delta}=1$, the optimization problem in Theorem 4 was found to be unfeasible, with $\gamma=1$. It was found to be feasible for all $\bar{\delta} \leq 0.648$. Recall that, for a free parameter $\gamma$, the optimization problem was found to be feasible for all $\bar{\delta}<2$. This fact shows that, using a free parameter $\gamma$ in the definition of the attractive invariant ellipsoid $\varepsilon(\mathbf{P}, \gamma)$, that is, the set (21), instead of fixing $\gamma=1$, gives less conservative stability conditions. Moreover, from Table 5 and for the case $u_{\max }=10$ and $\bar{\delta}=10$, the optimization problem with $\gamma=1$ is found to be feasible, as in Table 4. Nevertheless, the largest ellipsoid $\varepsilon_{\star}^{\prime}\left(\mathbf{P}_{\star}\right)$ obtained for $\gamma=1$, is too small compared with that obtained using a free parameter $\gamma$, that is, the ellipsoid $\varepsilon_{\star}\left(\mathbf{P}_{\star}, \gamma_{\star}\right)$. Recall that the largeness of the attractive invariant ellipsoid is measured by the "trace" function as noted in Tables 4 and 5. As noted in Table 4, for a free parameter $\gamma$, the volume of the largest ellipsoid was found to be about 46.5870. However, for a fixed parameter $\gamma=1$, the volume of the largest ellipsoid $\varepsilon_{\star}^{\prime}\left(\mathbf{P}_{\star}\right)$ is about 7.3840. The difference between the two sizes of the ellipsoid is evident. Another attractive result that can be observed from Table 5 is that the obtained matrix gain $\mathbf{K}_{\star}$ is too large compared with that obtained in Table 4.

Accordingly, we emphasize that the parameter $\gamma$ in the definition of the attractive ellipsoid (21) leads to less conservative results and then less restrictive LMI stability conditions. In addition, it contributes in obtaining a largest ellipsoid with a controller gain having a small size. Hence, the choice of $\gamma \neq 0$ reduces the conservatism of the LMIbased optimization problem in Theorem 4 .

Remark 9. We noted in the end of Section 4, just before Remark 5, that the computation time for solving the LMIbased optimization problem in Theorem 4 and then for the identification of the largest invariant attractive ellipsoid $\varepsilon_{\star}\left(\mathbf{P}_{\star}, \gamma_{\star}\right)$ depends on the number $N_{\kappa}$ of uniformly distributed points $\kappa$ in the interval ]0,1[ and the simulation time $t_{\kappa}$ for each value of the parameter $\kappa$. The total simulation time $t_{c}$ can be computed to be about $t_{c} \approx N_{\kappa} \times t_{\kappa}$. Tables 6 and 7 present the simulation results for different values of the parameters $N_{\kappa}$ for $u_{\max }=1$ (Table 6) and for $u_{\max }=10$ (Table 7). Recall that, for $u_{\max }=1$, we have $\bar{\delta}=1$, whereas for $u_{\max }=10$, we fixed $\bar{\delta}=10$. Moreover, the maximum bound $\rho$ of the disturbance is fixed to be $\rho=10$ in the two cases. Actually, in order to obtain these results in Tables 6 and 7, we have fixed the interval in which the parameter $\kappa$ varies as follows: [0.01: $\delta_{\kappa}: 0.99$ ], where we have selected four cases: $\delta_{\kappa}=0.01, \delta_{\kappa}=0.001, \delta_{\kappa}=0.0005$, and $\delta_{\kappa}=0.0002$. For these values of $\delta_{\kappa}$, it corresponds, respectively, the number $N_{\kappa}=99, N_{\kappa}=981, N_{\kappa}=1961$, and $N_{\kappa}=4901$. Thus, using these intervals and these values of the parameters $N_{\kappa}$, we simulated the optimization problem under LMI constraints in Theorem 4 and then we obtained the results in Tables 6 and 7. In these Tables, we give the two optimal gains, $\mathbf{K}_{\star}$ and $m_{\star}$, of the controller, $\lambda_{\star}$ (notice that $\lambda=\kappa /(1-\kappa)), \gamma_{\star}$, the size of the ellipsoid (trace $\left(\gamma_{\star} \mathbf{S}_{\star}\right)$ ), the simulation/computation time $t_{c}$, and the number of feasible solutions $N_{\mathrm{fs}}$. It is important to note that not all the $N_{\kappa}$-cases are feasible. From the results in Tables 6 and 7 , it reveals that the number of feasible solutions is only about $4.8 \%$ for $u_{\max }=1$ and about $6 \%$ for $u_{\max }=10$. The computation time $t_{c}$ for finding the largest ellipsoid increases significantly as $N_{\kappa}$ increases too. We also stress that as $N_{\kappa}$ increases, the optimal solution converges to that already obtained in Table 4 . We found the same values for $N_{\kappa}=4901$. Nevertheless, in this case, the computation time 
TABLe 5: Numerical results using Theorem 4 with a fixed parameter $\gamma=1$ and for $u_{\max }=1$ and $u_{\max }=10$.

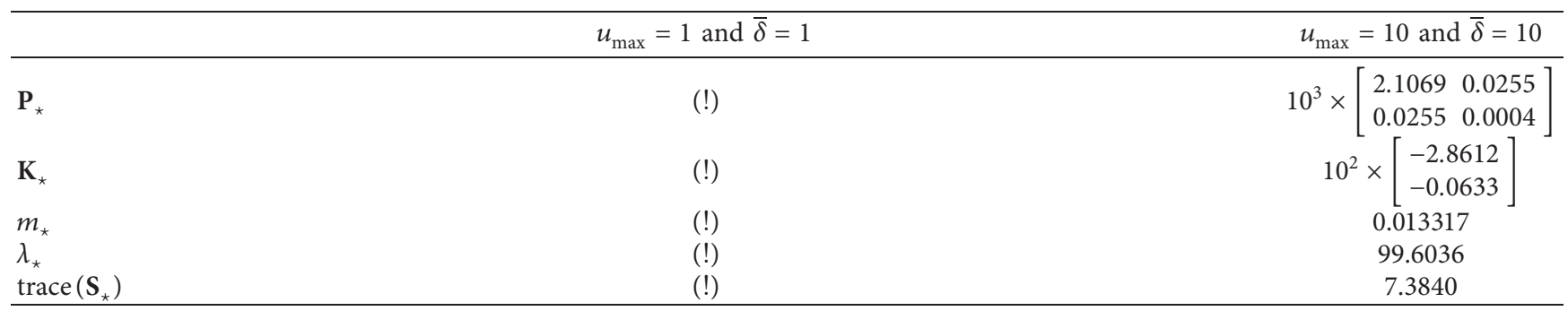

TABLe 6: Computation time for solving the LMI-based optimization algorithm in Theorem 4 for the case $u_{\max }=1$.

\begin{tabular}{|c|c|c|c|c|}
\hline$N_{\kappa}$ & 99 & 981 & 1961 & 4901 \\
\hline $\mathbf{K}_{\star}^{T}$ & {$\left[\begin{array}{c}-10.5679 \\
-1.1050\end{array}\right]$} & {$\left[\begin{array}{l}-9.2061 \\
-1.0321\end{array}\right]$} & {$\left[\begin{array}{l}-9.1147 \\
-1.0271\end{array}\right]$} & {$\left[\begin{array}{l}-9.0604 \\
-1.0242\end{array}\right.$} \\
\hline$m_{\star}$ & 0.013238 & 0.013237 & 0.013239 & 0.013235 \\
\hline$\lambda_{\star}$ & 19.0000 & 17.1818 & 17.0180 & 16.9211 \\
\hline$\gamma_{\star}$ & 0.0519 & 0.0574 & 0.0580 & 0.0583 \\
\hline $\operatorname{trace}\left(\gamma_{\star} \mathbf{S}_{\star}\right)$ & 1.6557 & 1.7874 & 1.7894 & 1.7906 \\
\hline$t_{c}[s]$ & 6.1988 & 54.9516 & 106.1182 & 264.1296 \\
\hline$N_{\mathrm{fs}}$ & 5 & 48 & 95 & 238 \\
\hline
\end{tabular}

TABLE 7: Computation time for solving the LMI-based optimization algorithm in Theorem 4 for the case $u_{\max }=10$.

\begin{tabular}{|c|c|c|c|c|}
\hline$N_{\kappa}$ & 99 & 981 & 1961 & 4901 \\
\hline $\mathbf{K}^{T}$ & {$[-18.6484]$} & {$[-19.7871]$} & {$[-20.3059$} & {$[-20.3362$} \\
\hline $\mathbf{K}_{\star}^{\star}$ & -1.8069 & -1.8309 & -1.8453 & -1.8458 \\
\hline$m_{\star}$ & 0.013306 & 0.013311 & 0.013306 & 0.013303 \\
\hline$\lambda_{\star}$ & 15.6667 & 16.8571 & 17.3486 & 17.3824 \\
\hline$\gamma_{\star}$ & 6.3740 & 5.9236 & 5.7557 & 5.7446 \\
\hline $\operatorname{trace}\left(\gamma_{\star} \mathbf{S}_{\star}\right)$ & 45.7911 & 46.5153 & 46.5625 & 46.5870 \\
\hline$t_{c}[s]$ & 3.1946 & 27.3301 & 53.4916 & 132.4823 \\
\hline$N_{\mathrm{fs}}$ & 6 & 59 & 117 & 293 \\
\hline
\end{tabular}

is very high, it is computed to be about 4.5 minutes for $u_{\max }=1$ and it is about 2.2 minutes for the second case $u_{\max }=10$.

5.3. Simulations for the Robust Stabilization of the Pitch Dynamics. In this part, we would show the robustness and effectiveness of the designed saturated affine state-feedback control law (19) in the robust stabilization of the uncertain disturbed nonlinear dynamics (15) of the pitch model of the helicopter. The control gains $\mathbf{K}$ and $m$ are provided in $\mathrm{Ta}-$ ble 4. We recall that, for $u_{\max }=1$, we have $\bar{\delta}_{a}=\bar{\delta}_{b}=\bar{\delta}_{c}=\bar{\delta}_{d}=\bar{\delta}_{e}=1$, whereas for $u_{\max }=10$, we take $\bar{\delta}_{a}=\bar{\delta}_{b}=\bar{\delta}_{c}=\bar{\delta}_{d}=\bar{\delta}_{e}=10$. Moreover, we recall that the external disturbance $w$ in the nonlinear dynamics (15) is bounded according to condition (14) with $\rho=10$. In addition, the uncertain parameters $\delta_{a}, \delta_{b}, \delta_{c}, \delta_{d}$, and $\delta_{e}$ in (15) vary randomly over time so that the boundedness condition (17) is satisfied. Furthermore, for the simulation of the controlled nonlinear system (15), we will take first an initial condition, $\mathbf{x}_{0}$, located outside the largest attractive ellipsoid $\varepsilon_{\star}\left(\mathbf{P}_{\star}, \gamma_{\star}\right)$ : for the case $u_{\max }=1$, we take $\mathbf{x}_{0}=\left[\begin{array}{ll}0.1 & -1\end{array}\right]^{\mathrm{T}}$, whereas for the case $u_{\max }=10$, we take $\mathbf{x}_{0}=\left[\begin{array}{ll}0.3 & -6\end{array}\right]^{\mathrm{T}}$.
Each initial condition is located near the boundary of the corresponding ellipsoid (see Figure 4). Furthermore, we will take an initial condition located outside the invariant at-

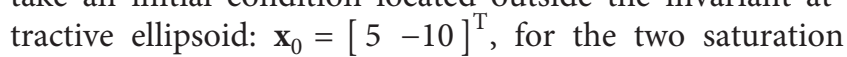
limits.

First of all, we will study the dynamics of the helicopter model under the saturated control law $u$ in the nominal case, that is, without parametric uncertainties and disturbance. Figure 5(a) shows temporal evolution of the states $x_{1}$ and $x_{2}$ of the certain undisturbed controlled nonlinear system (15), and Figure 5(b) reveals the saturated affine state-feedback control law $u$ for the saturation limit $u_{\max }=1$. It is obvious that the state of the pitch dynamics converges to zero, which is our main objective in this paper. Moreover, the control law $u$ converges to a constant value, which is found to be equal to the gain $m$. Actually, such constant value of the control input will ensure that the pitch dynamics of the helicopter stays at the zero state. In addition, we note that the reached maximum value of the control law $u$ is about 0.23 , which is less than the desired saturation limit $u_{\max }=1$.

We have also analyzed the nominal pitch dynamics of the helicopter model for the case $u_{\max }=10$. We have observed almost the same behavior in Figure 5. The reached 


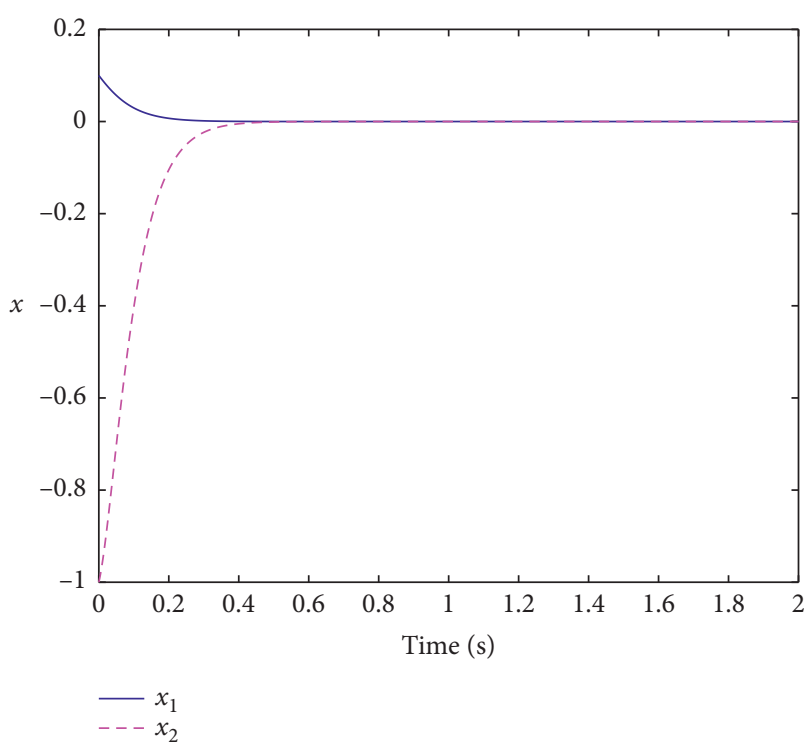

(a)

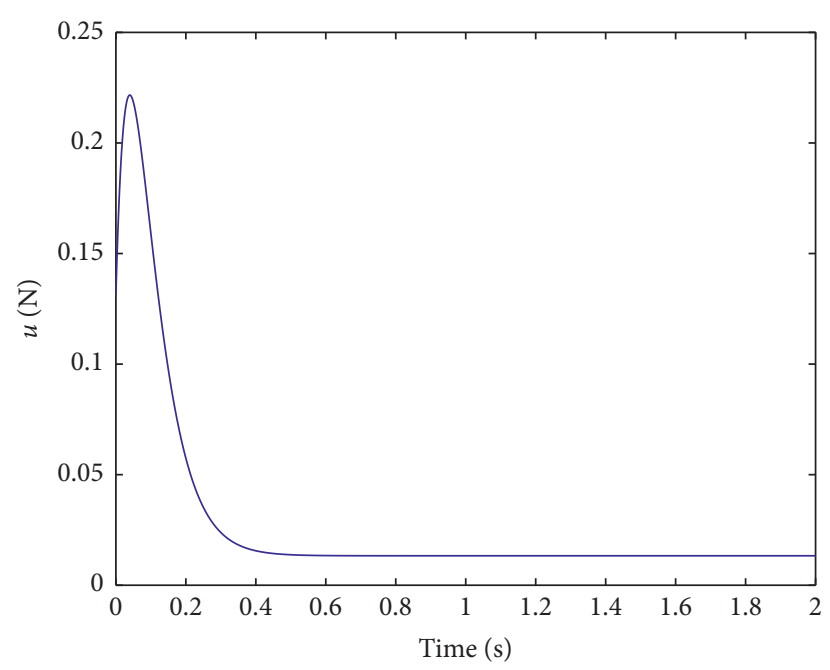

(b)

FIgURe 5: Evolution of the two states, $x_{1}$ and $x_{2}$, of the nonlinear system (15) and the saturated affine state-feedback control law $u$ for $u_{\max }=1$ in the nominal case. Here, the system is without uncertainties $\left(\delta_{a, b, c, d, e}=0\right)$ and disturbance $(w=0)$.

maximum value of the controller $u$ is around 5. The controller converges to the constant value $m \approx 0.01336$.

Now, we will show simulation results for the controlled nonlinear system subject to both parametric uncertainties and external disturbance. Simulation results are shown in Figure 6 for $u_{\max }=1$ and Figure 7 for $u_{\max }=10$. Figure 6(a) (resp. Figure 7(a)) presents temporal evolution of the two states, $x_{1}$ and $x_{2}$, of the nonlinear system (15) under uncertainties and disturbance. Figure 6(b) (resp. Figure 7(b)) reveals evolution of the saturated control law $u$ for $u_{\max }=1$ (resp. $u_{\max }=10$ ). As noted previously, the initial condition is located inside the largest attractive invariant ellipsoid. Moreover, the disturbance $w$ is injected into the system at $t=2[s]$ and during $3[s]$. We recall that the external disturbance $w$ satisfies condition (14) with $\rho=10$.

It is obvious from Figure 6(a) that the motion of the pitch dynamics of the helicopter model experiences some fluctuations around the desired zero state when the disturbance is applied. Once the effect of the external disturbing torque $w$ vanishes, the controlled system stabilizes again around its desired position with some very weak perturbations provoked by the injected parametric uncertainties. Moreover, in Figure 6(b), the control signal $u$ varies around the value $m=0.0133$. The control signal varies between two very small values: \pm 0.1 . We stress that the effects of the parametric uncertainties and the external disturbance are compensated. This shows the robustness of the control law $u$ towards the parametric uncertainties and the external disturbance with high amplitude.

However, for $u_{\max }=10$, the effect of the parametric uncertainties and the external disturbance $w$ is clear in Figure 7. Figure $7(a)$ is almost identical to Figure 6(a). Moreover, when the disturbance is injected, the control signal $u$ undergoes some fluctuations, which vary between \pm 1.5 . This happens because the maximum bound of the uncertainties is very large $\left(\bar{\delta}_{a, \ldots, e}=10\right)$ in this case compared with the first case, i.e., for $u_{\max }=1$.

It is worth to note that, in the previous three cases, the saturation level $\pm u_{\max }$ of the controller was well respected.

We have tested another case by taking an initial condition located outside the largest attractive invariant ellipsoid $\varepsilon_{\star}\left(\mathbf{P}_{\star}, \gamma_{\star}\right)$. We take here only the case $u_{\max }=1$, and then the corresponding largest ellipsoid is given by Figure 4(a). As noted previously, the initial condition is chosen to be $\mathbf{x}_{0}=[5-10]^{\mathrm{T}}$. Obtained results are illustrated in Figure 8. We observe first from Figure 8(a) that the state $x_{1}$ of the helicopter model experiences some smooth oscillations before its convergence and stabilization at the zero position despite the presence of the external disturbance and the parametric uncertainties. In fact, only weak fluctuations are observed as in Figure 7(a). However, the interesting phenomenon observed here is the saturation of the control law $u$ depicted in Figure 8(b).

Remark 10. It is worth to note that, for a predefined set of the system parameters, the previous established optimization problems under LMI constraints are (should be) simulated offline in order to compute the largest attractive ellipsoid and then the associated feedback gains $\mathbf{K}$ and $m$ of the input-saturated affine state-feedback controller $u$ before its application into a real helicopter system in practice. Thus, for a prescribed saturation level $u_{\max }$, the possible maximum allowable bounds of the parametric uncertainties $\bar{\delta}_{a}, \bar{\delta}_{b}, \bar{\delta}_{c}, \bar{\delta}_{d}$, and $\bar{\delta}_{e}$ and the possible maximum bound of the external disturbance signal $w$, i.e., $\rho$, we look, as described previously, for the largest attractive invariant ellipsoid $\varepsilon_{\star}\left(\mathbf{P}_{\star}, \gamma_{\star}\right)$. Thus, once this set is found, the corresponding feedback gains $\mathbf{K}$ and $m$ will be used in order to stabilize the pitch dynamics of the simple helicopter model in a real-world application. 


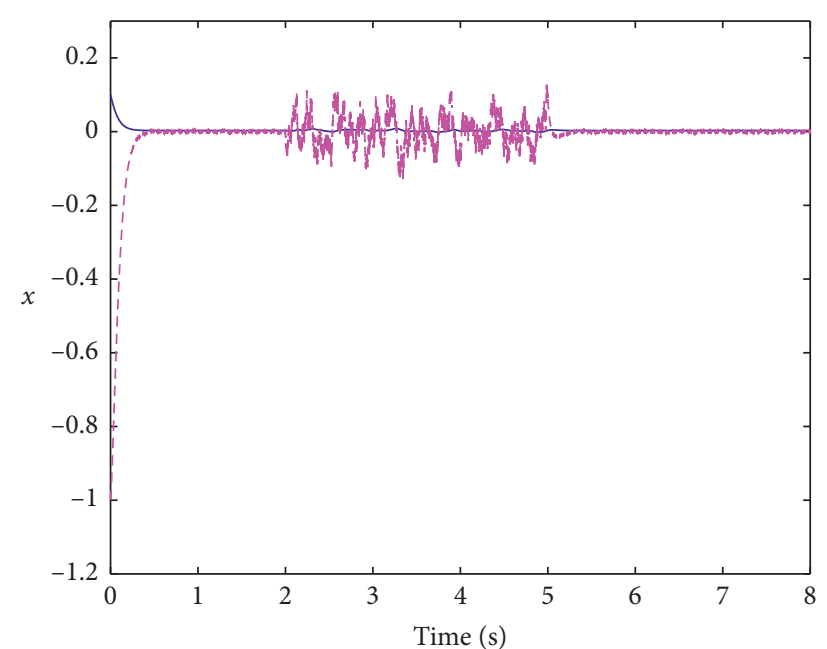

$\begin{aligned}- & x_{1} \\ --- & x_{2}\end{aligned}$

(a)

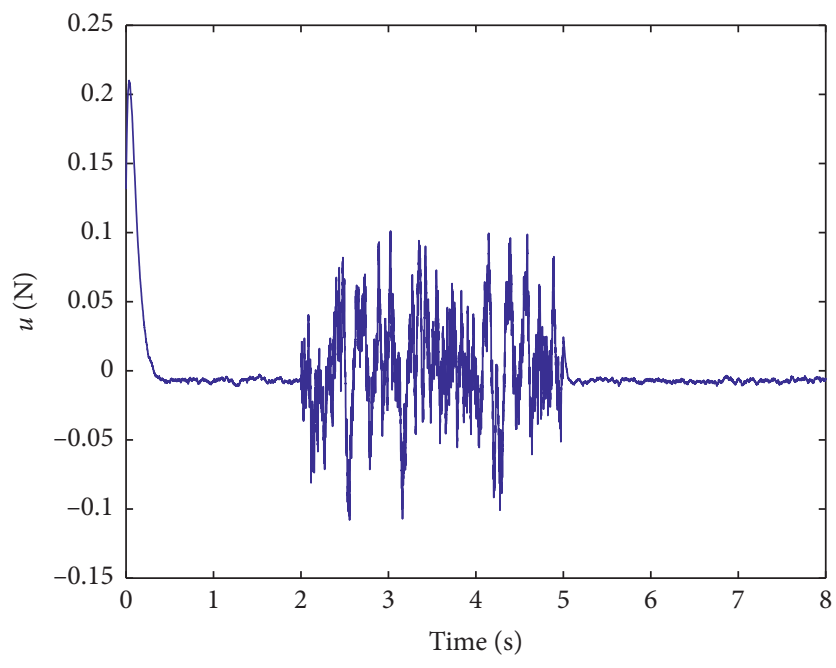

(b)

Figure 6: Temporal evolution of the states $x_{1}$ and $x_{2}$ of the nonlinear system (15) and the saturated affine state-feedback control law $u$ for the saturation level $u_{\max }=1$. Here, the system is subject to randomly time-varying uncertainties and also a randomly time-varying external disturbing torque $w$. Moreover, $\bar{\delta}_{a, b, c, d, e}=1$.

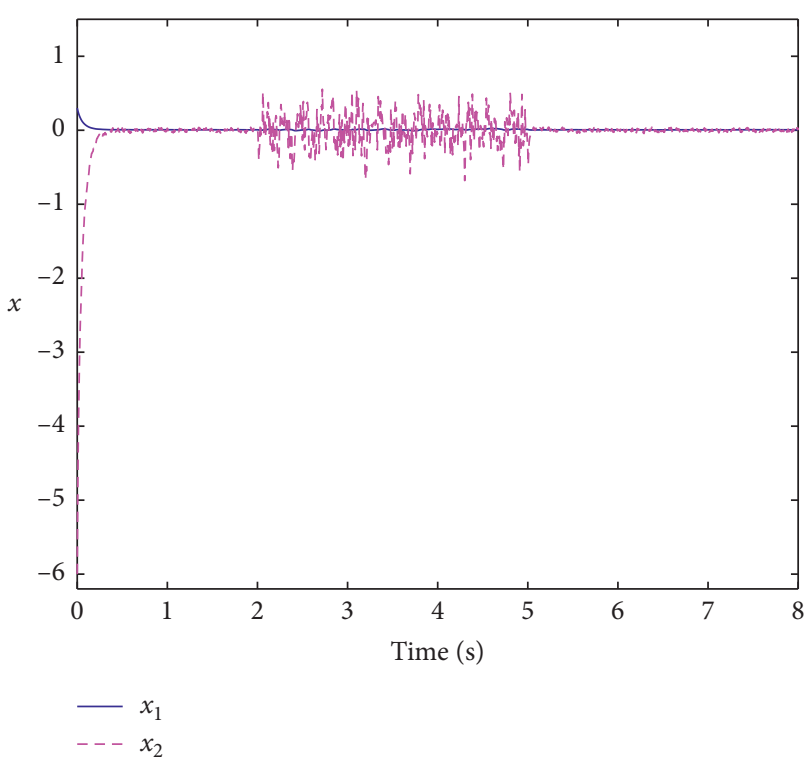

(a)

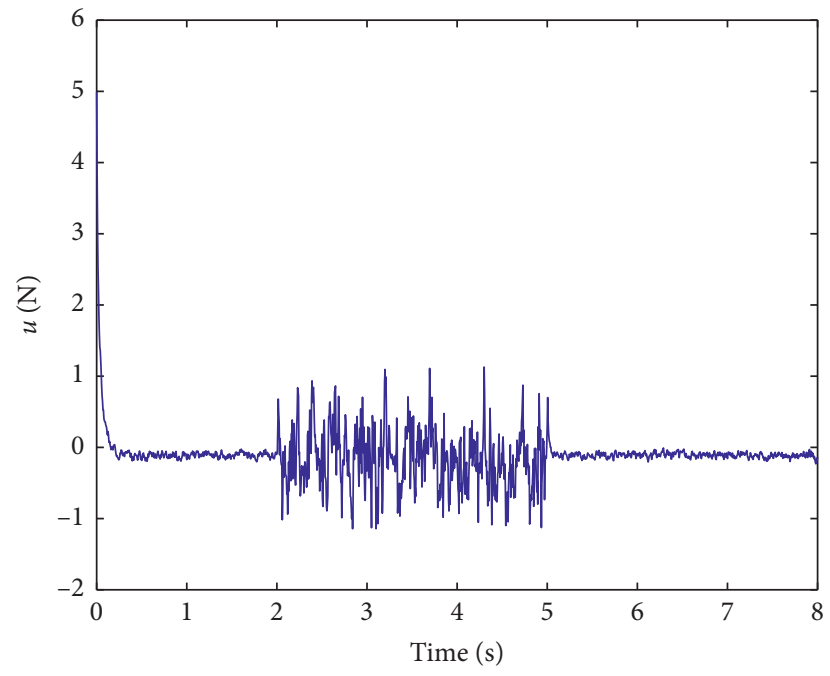

(b)

Figure 7: Evolution of the two states $x_{1}$ and $x_{2}$ of the nonlinear system (15) (a) and the saturated affine state-feedback control law $u$ (b) for the saturation level $u_{\max }=10$. In this case, we have $\bar{\delta}_{a, b, c, d, e}=10$.

\section{Conclusion and Future Works}

In this paper, an LMI-based approach for designing a robust affine state-feedback control law to stabilize the pitch dynamics of a helicopter model was proposed. The nonlinear dynamics of the helicopter was subject to an external disturbance and norm-bounded parametric uncertainties. Moreover, the problem of the actuator saturation in the design of the control law was as well addressed. We showed that the stabilization problem is represented as a solving problem of BMI constraints. Furthermore, with a judicious utilization of the Schur lemma and the matrix inversion lemma, these BMIs were transformed into LMIs. We have also developed an optimization problem with enhanced LMI constraints permitting to compute the maximum bounds of the parametric uncertainties.

In addition, we have proposed an LMI-based approach for the maximization of the attractive invariant ellipsoid for the uncertain disturbed nonlinear dynamics of the helicopter model under the saturated affine state-feedback control law. 


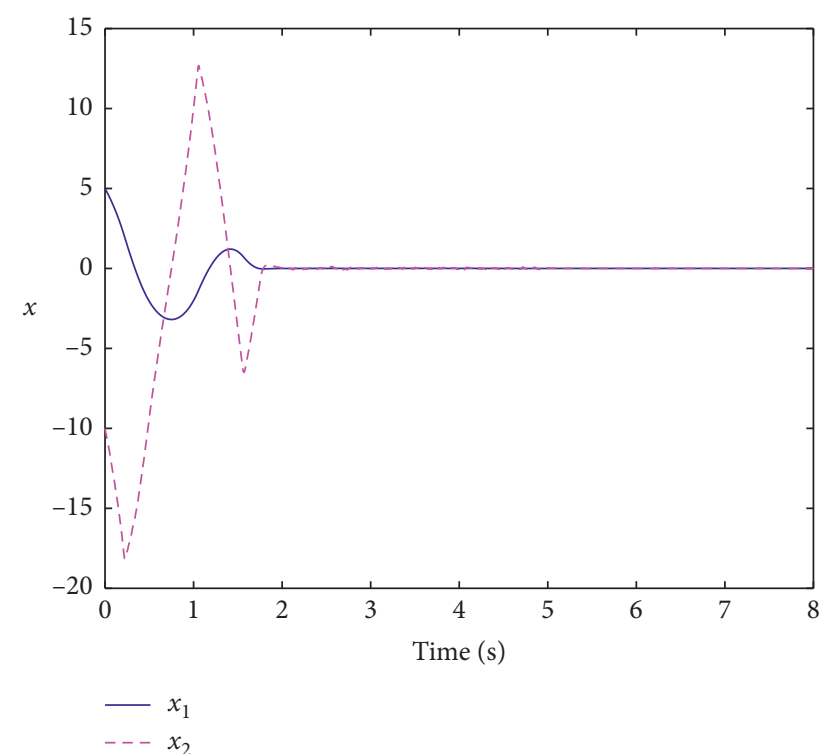

(a)

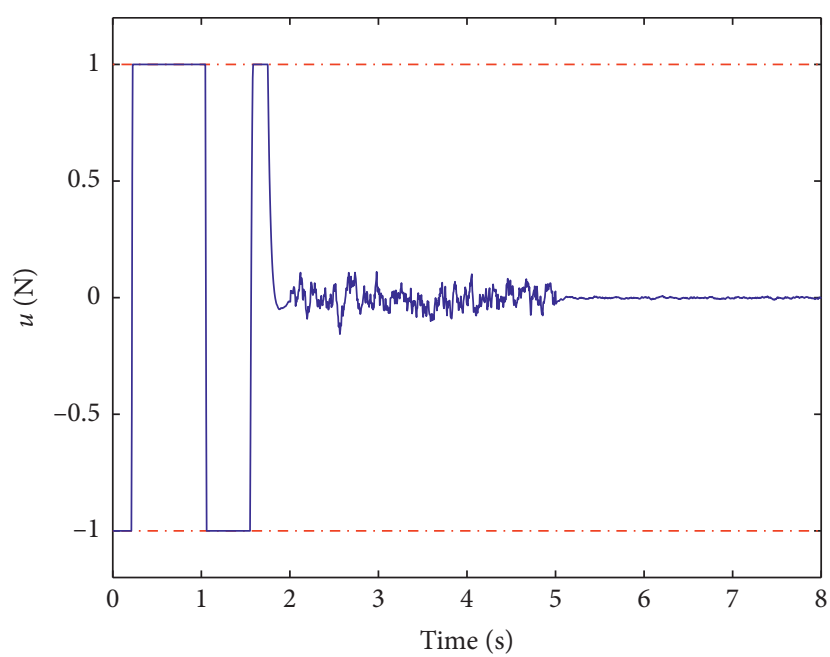

(b)

Figure 8: Temporal evolution of the two states $x_{1}$ and $x_{2}$ of the uncertain disturbed nonlinear system (15) and the saturated affine statefeedback control law $u$ for the saturation level $u_{\max }=1$. Here, the initial condition is selected to be outside the largest attractive invariant ellipsoid $\varepsilon_{\star}\left(\mathbf{P}_{\star}, \gamma_{\star}\right)$ in Figure $4(\mathrm{a})$.

The largeness of the ellipsoid was measured by means of the length of its semiaxis. We have showed that the optimization problem computes efficiently the largest ellipsoid in only one step.

Finally, we have showed through numerical simulations the performance of the synthesized saturated controller in the robust stabilization of the nonlinear dynamics under the norm-bounded parametric uncertainties and the external disturbing torque.

In the literature, the estimation of the domain of attraction of the closed-loop system subject to actuation saturations is one of important issues and several methods have been achieved for both linear and nonlinear systems. The difference between these methods lies in the approach by which the saturation nonlinearity was handled [51]. Its treatment can be classified into three main approaches: (1) the first one is to treat the actuator saturation problem as achieved in the present work, (2) the second approach is to treat it as a locally/generalized sector bounded nonlinearity $[17,69,70]$, (3) while the third method is to represent the saturation nonlinearity as a (polytopic or linear) differential inclusion (see $[36,51]$ and references insides). As noted in Remark 5, the solution presented in this work leads to the computation of the largest attractive invariant ellipsoid in only one step, compared to some related approaches that used a two-step method. Thus, the approach presented in this paper is more simpler and is less restrictive.

In addition, it worth to note that, in this paper, a saturated affine state-feedback controller was designed. To the best of our knowledge, such problem has not been considered in the literature. Generally, a saturated linear feedback controller has been considered to stabilize linear and nonlinear systems. Then, in order to realize a comparison and hence to show the possible efficiency of our design approach, a future work of this paper is the design of a saturated affine feedback controller using the sector-bounded nonlinearity method and the differential inclusion approach.

Furthermore, we aim at extending the present methodology of the saturation affine feedback controller for more complex nonlinear systems with different Lipschitzian conditions [65], and with measurement delays [71, 72] and also for the design of observer-based feedback controllers for Lipschitz nonlinear systems [3, 8, 64]. Moreover, we hope to extend this work for impulsive hybrid nonlinear systems, such as the biped robots [73] and the impact mechanical oscillators subject to multiple rigid constraints [74-76].

In the present work, we considered the simple pitch dynamics of a helicopter model as an application. Moreover, the zero state is not the equilibrium point of such model. Thus, we have designed an affine feedback controller under saturation to achieve the robust stabilization at the zero state. Another important application that has such feature is the robot manipulators [77]. Indeed, because of the gravitational matrix, the dynamics of robot manipulators has an equilibrium point different to the zero state. Thus, several control approaches have been adopted for this subject [77]. Our objective is then to extend the design method of the saturated affine state-feedback controller for the case of manipulator robots.

\section{Data Availability}

The data used to support the findings of this study are available from the corresponding author upon request.

\section{Conflicts of Interest}

The authors declare that there are no conflicts of interest regarding the publication of this paper. 


\section{References}

[1] M. G. Safonov, "Origins of robust control: early history and future speculations," Annual Reviews in Control, vol. 36, no. 2, pp. 173-181, 2012.

[2] M. S. Sadabadi and D. Peaucelle, "From static output feedback to structured robust static output feedback: a survey," Annual Reviews in Control, vol. 42, pp. 11-26, 2016.

[3] S. Ahmad, M. Rehan, and K.-S. Hong, "Observer-based robust control of one-sided Lipschitz nonlinear systems," ISA Transactions, vol. 65, pp. 230-240, 2016.

[4] I. R. Petersen and R. Tempo, "Robust control of uncertain systems: classical results and recent developments," Automatica, vol. 50, no. 5, pp. 1315-1335, 2014.

[5] A. Poznyak, A. Polyakov, and V. Azhmyakov, Attractive Ellipsoids in Robust Control, Systems \& Control: Foundations \& Applications, Birkhauser Basel, Basel, Switzerland, 1st edition, 2014.

[6] R. K. Yedavalli, Robust Control of Uncertain Dynamic Systems. A Linear State Space Approach, Springer, Berlin, Germany, 1st edition, 2014

[7] B. El Haiek, H. El Aiss, H. Abdelaziz, A. El Hajjaji, and T. El Houssaine, "New approach to robust observer-based control of one-sided Lipschitz non-linear systems," IET Control Theory \& Applications, vol. 13, no. 9, pp. 333-342, 2019.

[8] M. Rehan, S. Ahmad, and K.-S. Hong, "Novel results on observer-based control of one-sided Lipschitz systems under input saturation," European Journal of Control, 2019.

[9] S. Boyd, L. El-Ghaoui, E. Feron, and V. Balakrishnan, Linear Matrix Inequalities in System and Control Theory, Vol. 15 of Studies in Applied and Numerical Mathematics, Society for Industrial and Applied Mathematics (SIAM), Philadelphia, PA, USA, 1st edition, 1994.

[10] J. Lofberg, "YALMIP: a toolbox for modeling and optimization in MATLAB," in Proceedings of the IEEE International Conference on Robotics and Automation, pp. 284-289, New Orleans, LA, USA, September 2004.

[11] A. Zemouche, R. Rajamani, H. Kheloufi, and F. Bedouhene, "Robust observer-based stabilization of Lipschitz nonlinear uncertain systems via LMIs-discussions and new design procedure," International Journal of Robust and Nonlinear Control, vol. 27, no. 11, pp. 1915-1939, 2017.

[12] H. Gritli and S. Belghith, "Robust feedback control of the underactuated inertia wheel inverted pendulum under parametric uncertainties and subject to external disturbances: LMI formulation," Journal of the Franklin Institute, vol. 355, no. 18, pp. 9150-9191, 2018.

[13] Y. Wang, R. Rajamani, and A. Zemouche, "Sequential LMI approach for the design of a BMI-based robust observer state feedback controller with nonlinear uncertainties," International Journal of Robust and Nonlinear Control, vol. 28, no. 4, pp. 1246-1260, 2018.

[14] A. Aktas, H. Yazici, and M. Sever, "LMI-based design of an I-PD+PD type LPV state feedback controller for a gantry crane," Transactions of the Institute of Measurement and Control, vol. 41, no. 6, pp. 1640-1655, 2019.

[15] S. M. Moradi, A. Akbari, and M. Mirzaei, "An offline LMIbased robust model predictive control of vehicle active suspension system with parameter uncertainty," Transactions of the Institute of Measurement and Control, vol. 41, no. 6, pp. 1699-1711, 2019.

[16] I. H. Brahim, D. Mehdi, and M. Chaabane, "Sensor fault and state estimation for uncertain fuzzy descriptor systems: an
LMI approach," Transactions of the Institute of Measurement and Control, vol. 41, no. 1, pp. 135-144, 2019.

[17] S. Tarbouriech, G. Garcia, J. M. Gomes da Silva Jr., and I. Queinnec, Stability and Stabilization of Linear Systems with Saturating Actuators, Springer, Berlin, Germany, 1st edition, 2011.

[18] T. Hu and Z. Lin, Control Systems with Actuator Saturation: Analysis and Design (Control Engineering), Birkhauser Basel, Basel, Switzerland, 1st edition, 2011.

[19] M. L. Corradini, A. Cristofaro, F. Giannoni, and G. Orlando, Control Systems with Saturating Inputs. Analysis Tools and Advanced Design, Lecture Notes in Control and Information Sciences, Springer, Berlin, Germany, 1st edition, 2012.

[20] T. Hu and Z. Lin, "Exact characterization of invariant ellipsoids for single input linear systems subject to actuator saturation," IEEE Transactions on Automatic Control, vol. 47, no. 1, pp. 164-169, 2002.

[21] T. Alamo, A. Cepeda, and D. Limon, "Improved computation of ellipsoidal invariant sets for saturated control systems," in Proceedings of the 44th IEEE Conference on Decision and Control, pp. 6216-6221, Seville, Spain, December 2005.

[22] Q. Wang, "Global external stochastic stabilization of linear systems with input saturation: an alternative approach," Complexity, vol. 2017, Article ID 3517280, 7 pages, 2017.

[23] B. Zhou and G.-R. Duan, "Global stabilization of linear systems via bounded controls," Systems \& Control Letters, vol. 58, no. 1, pp. 54-61, 2009.

[24] P. Niamsup and V. N. Phat, "Robust finite-time control for linear time-varying delay systems with bounded control," Asian Journal of Control, vol. 18, no. 6, pp. 2317-2324, 2016.

[25] M. Ran, Q. Wang, and C. Dong, "Anti-windup design for uncertain nonlinear systems subject to actuator saturation and external disturbance," International Journal of Robust and Nonlinear Control, vol. 26, no. 15, pp. 3421-3438, 2016.

[26] M. Ran, Q. Wang, and C. Dong, "Stabilization of a class of nonlinear systems with actuator saturation via active disturbance rejection control," Automatica, vol. 63, pp. 302-310, 2016.

[27] D. W. Kim, "Further refinement on controller design for linear systems with input saturation," Automatica, vol. 77, pp. 14-17, 2017.

[28] Y. Li and Z. Lin, "A complete characterization of the maximal contractively invariant ellipsoids of linear systems under saturated linear feedback," IEEE Transactions on Automatic Control, vol. 60, no. 1, pp. 179-185, 2015.

[29] Y. Li and Z. Lin, "The maximal contractively invariant ellipsoids for discrete-time linear systems under saturated linear feedback," Automatica, vol. 76, pp. 336-344, 2017.

[30] L. Amezquita-Brooks, D. Hernandez, F. Gonzalez-Sanchez, and J. C. Tudón-Martínez, "Linear programming predictive control with actuator saturation: experimental robustness and performance results," Proceedings of the Institution of Mechanical Engineers, Part I: Journal of Systems and Control Engineering, vol. 229, no. 8, pp. 700-710, 2015.

[31] T. Binazadeh and M. Bahmani, "Robust time-varying output tracking control in the presence of actuator saturation," Transactions of the Institute of Measurement and Control, vol. 40, no. 1, pp. 61-70, 2018.

[32] M. Nasri, D. Saifia, M. Chadli, and S. Labiod, "Ho static output feedback control for electrical power steering subject to actuator saturation via fuzzy Lyapunov functions," Transactions of the Institute of Measurement and Control, vol. 41, no. 12, pp. 3340-3351, 2019. 
[33] K. S. T. Alain, A. T. Azar, F. H. Bertrand, and K. Romanic, "Robust observer-based synchronisation of chaotic oscillators with structural perturbations and input nonlinearity," International Journal of Automation and Control, vol. 13, no. 4, pp. 387-412, 2019.

[34] R. Mei and C. Yu, "Adaptive neural output feedback control for uncertain robot manipulators with input saturation," Complexity, vol. 2017, Article ID 7413642, 12 pages, 2017.

[35] Q. Wang, M. Ran, and C. Dong, "An analysis and design method for a class of nonlinear systems with nested saturations," International Journal of Control, vol. 89, no. 8, pp. 1711-1724, 2016.

[36] Y. Li and Z. Lin, "Improvements to the linear differential inclusion approach to stability analysis of linear systems with saturated linear feedback," Automatica, vol. 49, no. 3, pp. 821-828, 2013.

[37] H. Du, N. Zhang, and G. Dong, "Stabilizing vehicle lateral dynamics with considerations of parameter uncertainties and control saturation through robust yaw control," IEEE Transactions on Vehicular Technology, vol. 59, no. 5, pp. 2593-2597, 2010.

[38] W. Sun, Z. Zhao, and H. Gao, "Saturated adaptive robust control for active suspension systems," IEEE Transactions on Industrial Electronics, vol. 60, no. 9, pp. 3889-3896, 2013.

[39] H. Du and N. Zhang, "Fuzzy control for nonlinear uncertain electrohydraulic active suspensions with input constraint," IEEE Transactions on Fuzzy Systems, vol. 17, no. 2, pp. 343$356,2009$.

[40] W. Sun, H. Gao, and O. Kaynak, "Vibration isolation for active suspensions with performance constraints and actuator saturation," IEEE/ASME Transactions on Mechatronics, vol. 20, no. 2, pp. 675-683, 2015.

[41] F. Zhao, S. S. Ge, M. Dong, F. Tu, and Y. Qin, "Adaptive neural network control for active suspension system with actuator saturation," IET Control Theory \& Applications, vol. 10, no. 14, pp. 1696-1705, 2016.

[42] K. Lu, Y. Xia, C. Yu, and H. Liu, "Finite-time tracking control of rigid spacecraft under actuator saturations and faults," IEEE Transactions on Automation Science and Engineering, vol. 13, no. 1, pp. 368-381, 2016.

[43] L. Sun and Z. Zheng, "Disturbance-observer-based robust backstepping attitude stabilization of spacecraft under input saturation and measurement uncertainty," IEEE Transactions on Industrial Electronics, vol. 64, no. 10, pp. 7994-8002, 2017.

[44] B. Qiang and L. Zhang, "Output feedback control design to enlarge the domain of attraction of a supercavitating vehicle subject to actuator saturation," Transactions of the Institute of Measurement and Control, vol. 40, no. 10, pp. 3189-3200, 2018.

[45] W. Chang, S. Tong, and Y. Li, “Adaptive fuzzy backstepping output constraint control of flexible manipulator with actuator saturation," Neural Computing and Applications, vol. 28, no. 1, pp. 1165-1175, 2017.

[46] Z. Zheng and L. Sun, "Path following control for marine surface vessel with uncertainties and input saturation," Neurocomputing, vol. 177, pp. 158-167, 2016.

[47] T. Hu, Z. Lin, and B. M. Chen, "An analysis and design method for linear systems subject to actuator saturation and disturbance," Automatica, vol. 38, no. 2, pp. 351-359, 2002.

[48] T. Hu, Z. Lin, and B. M. Chen, "Analysis and design for discrete-time linear systems subject to actuator saturation," Systems \& Control Letters, vol. 45, no. 2, pp. 97-112, 2002.

[49] Z. Zuo and Y. Wang, "On enlarging the domain of attraction for linear systems subject to actuator saturation,"
International Journal of General Systems, vol. 37, no. 2, pp. 239-248, 2008.

[50] B. Zhou, W. X. Zheng, and G.-R. Duan, "Stability and stabilization of discrete-time periodic linear systems with actuator saturation," Automatica, vol. 47, no. 8, pp. 1813-1820, 2011.

[51] B. Zhou, W. X. Zheng, and G.-R. Duan, "An improved treatment of saturation nonlinearity with its application to control of systems subject to nested saturation," Automatica, vol. 47, no. 2, pp. 306-315, 2011.

[52] T. Hu and Z. Lin, "On enlarging the basin of attraction for linear systems under saturated linear feedback," Systems \& Control Letters, vol. 40, no. 1, pp. 59-69, 2000.

[53] Y. Chen, Q. Zhou, and S. Fei, "Robust stabilization and 12 gain control of uncertain discrete-time constrained piecewiseaffine systems," Nonlinear Dynamics, vol. 75, no. 1-2, pp. 127-140, 2014

[54] N. Vafamand, M. H. Asemani, and A. Khayatiyan, "A robust L1 controller design for continuous-time TS systems with persistent bounded disturbance and actuator saturation," Engineering Applications of Artificial Intelligence, vol. 56, pp. 212-221, 2016.

[55] B. Samadi and L. Rodrigues, "Stability of sampled-data piecewise affine systems: a time-delay approach," Automatica, vol. 45, no. 9, pp. 1995-2001, 2009.

[56] K. Merat, H. Salarieh, A. Alasty, and A. Meghdari, "Stochastic piecewise affine control with application to pitch control of helicopter," Nonlinear Analysis: Hybrid Systems, vol. 15, pp. 86-97, 2015.

[57] H. Razavi, K. Merat, H. Salarieh, A. Alasty, and A. Meghdari, "Observer based minimum variance control of uncertain piecewise affine systems subject to additive noise," Nonlinear Analysis: Hybrid Systems, vol. 19, pp. 153-167, 2016.

[58] F. Mesquine, H. Ayad, and M. Ait Rami, "Disturbance attenuation for continuous-time linear systems with state and control constraints," International Journal of Sciences and Techniques of Automatic Control \& Computer Engineering Special Issue CSC'07, pp. 312-324, 2007.

[59] M. Rehan and K.-S. Hong, "Decoupled-architecture-based nonlinear anti-windup design for a class of nonlinear systems," Nonlinear Dynamics, vol. 73, no. 3, pp. 1955-1967, 2013.

[60] M. Rehan, M. Tufail, C. K. Ahn, and M. Chadli, "Stabilisation of locally Lipschitz non-linear systems under input saturation and quantisation," IET Control Theory \& Applications, vol. 11, no. 9, pp. 1459-1466, 2017.

[61] S. Krafes, Z. Chalh, and A. Saka, "A review on the control of second order underactuated mechanical systems," Complexity, vol. 2018, Article ID 9573514, 17 pages, 2018.

[62] Y. Liu and H. Yu, "A survey of underactuated mechanical systems," IET Control Theory \& Applications, vol. 7, no. 7, pp. 921-935, 2013.

[63] W. Zhang, H.-S. Su, Y. Liang, and Z.-Z. Han, "Non-linear observer design for one-sided Lipschitz systems: an linear matrix inequality approach," IET Control Theory \& Applications, vol. 6, no. 9, pp. 1297-1303, 2012.

[64] S. Ahmad and M. Rehan, "On observer-based control of onesided Lipschitz systems," Journal of the Franklin Institute, vol. 353, no. 4, pp. 903-916, 2016.

[65] H. Gritli and S. Belghith, "LMI-based design of state feedback controller for Lipschitzian nonlinear systems," in Proceedings of the 15th International Multi-Conference on Systems, Signals \& Devices (SSD), pp. 306-314, Hammamet, Tunisia, March 2018. 
[66] C. Venkatesan, "Fundamentals of helicopter dynamics," in Engineering \& Technology, CRC Press, Boca Raton, FL, USA, 1st edition, 2014.

[67] I. A. Raptis and K. P. Valavanis, Linear and Nonlinear Control of Small-Scale Unmanned Helicopters, Vol. 45 of Intelligent Systems, Control and Automation: Science and Engineering, Springer, Berlin, Germany, 1st edition, 2011.

[68] H. Kheloufi, A. Zemouche, F. Bedouhene, and M. Boutayeb, "On LMI conditions to design observer-based controllers for linear systems with parameter uncertainties," Automatica, vol. 49, no. 12, pp. 3700-3704, 2013.

[69] Y. Chen, Y. Li, and S. Fei, "Anti-windup design for time-delay systems via generalised delay-dependent sector conditions," IET Control Theory \& Applications, vol. 11, no. 10, pp. 1634-1641, 2017.

[70] Y. Chen, Z. Wang, S. Fei, and Q.-L. Han, "Regional stabilization for discrete time-delay systems with actuator saturations via a delay-dependent polytopic approach," IEEE Transactions on Automatic Control, vol. 64, no. 3, pp. 12571264, 2019.

[71] S. Ahmad, R. Majeed, K.-S. Hong, and M. Rehan, "Observer design for one-sided Lipschitz nonlinear systems subject to measurement delays," Mathematical Problems in Engineering, vol. 2015, Article ID 879492, 13 pages, 2015.

[72] S. Ahmad, M. Rehan, and M. Iqbal, "Robust generalized filtering of uncertain Lipschitz nonlinear systems under measurement delays," Nonlinear Dynamics, vol. 92, no. 4, pp. 1567-1582, 2018.

[73] H. Gritli and S. Belghith, "Walking dynamics of the passive compass-gait model under OGY-based control: emergence of bifurcations and chaos," Communications in Nonlinear Science and Numerical Simulation, vol. 47, pp. 308-327, 2017.

[74] H. Gritli and S. Belghith, "Diversity in the nonlinear dynamic behavior of a one-degree-of-freedom impact mechanical oscillator under OGY-based state-feedback control law: order, chaos and exhibition of the border-collision bifurcation," Mechanism and Machine Theory, vol. 124, pp. 1-41, 2018.

[75] H. Gritli, "Robust master-slave synchronization of chaos in a one-sided 1-DoF impact mechanical oscillator subject to parametric uncertainties and disturbances," Mechanism and Machine Theory, vol. 142, p. 103610, 2019.

[76] F. Turki, H. Gritli, and S. Belghith, "An LMI-based design of a robust state-feedback control for the master-slave tracking of an impact mechanical oscillator with double-side rigid constraints and subject to bounded-parametric uncertainty," Communications in Nonlinear Science and Numerical Simulation, vol. 82, p. 105020, 2020.

[77] R. Kelly, V. Santibáñez Davila, and J. A. Loría Perez, Control of Robot Manipulators in Joint Space (Advanced Textbooks in Control and Signal Processing), Springer, Berlin, Germany, 1st edition, 2005. 